XXXV.

\title{
30. Wanderversammlung der Siidwestdeutschen Neurologen und Irrenärzte in Baden-Baden am 27. und 28. Mai 1905 .
}

Anwesend sind die Herren:

Privatdocent Dr. Arnsperger (Heidelberg), Dr. S. Auerbach (Franlffurt a. M.), Dr. Aumüller (Stephansfeld), Prof. Dr. Axenfeld (Freiburg), Med.-Rat Dr. Barbo (Pforzheim), Dr. Barth (Baden-Baden), Geh.-Rath Prof. Bäumler (Freiburg), Dr. Bayerthal (Worms), Dr. Becker (Baden-Baden), Dr. Belzer (BadenBaden), Dr. Berliner (Frankfurt a. M.), Privatdocent Dr. Bethe (Strassburg i. E.), Dr. E. Beyer (Littenweiler), Dr. Bischoff (Tübingen), Dr. Blachiau (Werneck), Dr. Bl um (Frankfurt a. M.), Prof. Dr. Brauer (Marburg), Privatdocent Dr. Bumke (Freiburg), Prof. Dr. Braus (Heidelberg), Dr. Brosius (Saarbrücken), Prof. Dr. Cohnheim (Heidelberg), Dr. Curschmann (Tübingen), Dr. Daiber (Weinsberg), Dr. Dambacher (Karlsruhe), Dr. Dammert (Baden-Baden), Dr. Damköhler (Klingenmünster), Dr. Dietz (Goddelau), Privatdocent Dr. Dreyfus (Strassburg), Dr. Dreyfuss (Heidelberg), Dr. Ebers (Baden-Baden), Prof. Dr. Edinger (Frankfurt a. M.), Dr. Ehrhardt (Winnenthal); Geh. Rath Prof. Erb (Heidelberg), Prof. Dr. Ewald (Strassburg), Med.-Rath Dr. Feldbausch (Emmendingen), Dr. Fischler (Heidelberg), Geheimrath Prof. Fleiner (Heidelberg), Dr. Friedländer (Wiesbaden), Dr. Friedmann (Mannbein), Dr. Fuchs (Emmendingen), Dr. Fürer (Rockenau), Dr. Fürnrohr (Berlin), Hofrath Prof. Dr. Fürstner (Strassburg), Privatdocent Dr. Gaupp (München), Hofrath Dr. Gilbert (Baden-Baden), Dr. Gross (Heidelberg), Med.-Rath. Dr. Haardt (Emmendingen), Dr. Haberkant (Stephansfeld), Dr. Hardt (Berlin), Dr. Heiligenthal (Baden-Baden), Dr. Herzog (Mainz), Dr. Herzog (Heidelberg), Dr. Hey (Strassburg), Prof. Dr. Hoche (Freiburg), Hofrath Dr. v. Hoffmann (Baden-Baden), Prof. Dr. A. Hofmann (Düsseldorf), Di. A. Homburger (Frankfurt a. M.), Dr. Horstmann, Dr. Homfeld (Klingenmünster), Dr. Hübner 
30. Wanderyers. der Südwestdeutschen Neurologen u. Irrenärzte. 1021

(Lichtenthal), Dr. Jederhorn (Strassburg), Dr. Jäger (Giessen), Privatdocent Dr. Jahrmärker (Marburg), Privatdocent Dr. Jamin (Erlangen), Privatdocent Dr. Ibrahim (Heidelberg), Prof. Dr. Jolgersma (Leiden), Dr. Igersheimer (Strassbarg), Dr. Jolosse (Hamburg), Dr. Jones (Freiburg), Dr. Kalberlah (Frankfurt a.M.), Dr. Fr. Ka ufmann (BadDürkheim), Prof. Dr. Kirch hoff (Sohleswig), Dr. Klaus (Freiburg), Prof. Dr. Krehl (Strassburg), Dr. Kress (Heppenheim), Med.-Rath Dr. Kürz (Heidelberg), Hofrath Dr. Landerer (Kennenburg), Geh.-Rath Prof. Leber (Heidelberg), Dr. L. Laquer (Frankfurt a. M.), Dr. Lilienstein (Bad Nauheim), Privatdocent Dr. Link (Freiburg), Dr. Longard (Heidelberg), Hofrath Prof. Dr. Lossen (Heidelberg), Prof. Dr. Magnus (Heidelberg), Dr. Mann (Ilannheim), Dr. Mauss (Coswig-Dresden), Dr. Meyer (Strassburg), Dr. Merzbacher (Heidelberg), Dr. de Mon tet (Frankfurt a. M.), Privatdocent Dr. E. Müller (Breslau), Dr. Rob. Müller (Strassburg), Geh.-Rath Prof. Naunyn (Baden-Baden), Dr. Neumann (Karlsruhe), Prof. Dr. Nissl (Heidelberg), Prof. Dr. Nolda (St. Moritz), Hofrath Dr. Obkireher (Baden-Baden), Dr. van Oordt (St. Blasien), Dr. Osann (Strassburg), Med.-Rath Dr. Oster (Illenau), Dr. Petri (Freiburg), Dr. Pfers d orf (Strassburg), Prof. Dr. Pfister (Freiburg), Dr. v. Rad (Nürnberg), Dr. Preis (Neckargemünd), Dr.Preis (Strassburg), Prof. Dr.v.Preuss (Wien), Dr. Riffel (Emmendingen), Dr. Rittershaus (Goddelar), Dr. Römer (Hirsau), Dr. Rosi (Paris), Prof. Dr. Schaffer (Budapest), San.-Rath Dr. Schliep (Baden-Baden), Dr. Schneider (Goddelau). Privatdoc. Dr. Schönborn (Heidelberg), Dr. Schridde (Erlangen), Dr. Schütz (Wiesbaden), Dr. Spielmeyer (Freiburg), Dr. Starck (Karlsruhe), Dr. Stengel (Bruchsal), Dr. Tobler (Heidelberg), Dr. Thoma (Illenau), Privatdoc. Dr. Volhard (Giessen), Dr. Wagner (Lindenburg-Cöln), Dr. Wallenberg (Danzig), Dr. M. Weil (Stuttgart), Professor Dr. Weintrand (Wiesbaden), Dr. Wertheimer (Mannheim), Professor Dr. Wollenberg (Tübingen), Dr. Zahn (Stuttgart).

Ihr Fernbleiben haben entschuldigt und die Versammlung begrüsst die

Dr. Albrecht (Frankfurt a. M.), Prof. Dr. A schaffenburg (Cöln), Privatdoc. Dr. Bartels (Marburg), Geh.-Rath Prof. Hitzig (Halle), Dr. Nonne (Hamburg), Geh.-Rath Prof. v. Leube (Würzburg), Prof. v. Monakow (Zürich), Prof. Specht (Tübingen), Gea.-Rath Prof. F. Schultze (Bonn), Geh.-R. Prof, v. Strümpell (Breslau), Prof. Schwalbe (Strassburg), Geh.-Rath Schüle (Illenau), Prof. Schäle (Freiburg), Hofrath Dr. Wurm (Teinach), Geh.-Rath Prof. Ziehen (Berlin). 
1022 30. Wandervers. der Südwestdeutschdn Neurologen u. Irrenärzte.

I. Sitzung: Sonnabend, 27. Mai 1905, Vormittags 11 Uhr.

Vorsitzender: Herr Geheimrath Erb (Heidelberg).

Schriftührer: Privatdocent Dr. Bumke (Freiburg) und

Dr. A. Homburger (Frankfurt a. M.).

Herr Edinger (Frankfurt a. M.) begrüsst als Geschäftsführer die Versammlung. Er theilt zunächst mit, dass der andere Geschäftsführer, Herr Fischer-Pforzheim leider durch Unwohlsein verhindert ist, der Versammlung beizuwohnen; die Geschäftsführer haben Herrn Geh. Rath Ludwig-Heppenheim, einem der Stifter der Versammlung zum 80. Geburtstag gratulirt und ein Dankschreiben empfangen.

Edinger gedenkt der Verstorbenen des Jahres: Eckhardt's, des Physiologen, der mit zu den Begründern der experimentellen Physiologie des Nervensystems gehört und CarI Weigert's.

"Wer immer in den letzten 20 Jahren mit der Anatomie des Nervensystems sich beschäftigt hat, der weiss, was er dem Manne schuldet, der seine beste Kraft an die Entdeckung, Ausarbeitung und Sicherung von Methoden gesetat hat. Weigert danken wir es, wenn wir heute imstande sind präcis zu schneiden, denn er hat mit Schanze die ersten exacten Mikrotome construirt; er hat uns gelehrt, dass in der Wärme die Fixirung schneller eintritt und hat die Chromhärtung und Beizung verbessert und abgekürzt. Weigert hat die Celloidinmethode erst brauchbar gemacht und hat sie zur exquisiten Serienmethode für die Behandlung grosser Schnitte ausgestaltet; ihm verdanken wir die Papierstreifentechnik des Auflegens, die serienweise Färbung der auf dem Objectträger zwischen zwei Celloidinschichten eingebeiteten Schnitte und deren Entwässerung und Aufhellung mit Carbolxylol. Vor allem aber hat Weigert zuerst gezeigt, dass man im Nervensystem electiv färben liann. Ehe er auf den Plan trat, batte man im Carmin und einigen Anilinfarben Körper, die nur der Intensität nach verschiedene Gewebe verschieden tingirten, jede Färbung war eine Art Kunststück und ihre Deutung war es oft noch mehr. In harter Arbeit, bei der er sich nie genug thun konnte, hat We ige r t Methoden erfunden, welche gestatten, markhaltigen Nervenfasern electì zu färben, eine Methode, welche die Glia allein tingirt, ebenso wie er als Vermächtniss uns noch eine Kernfärbung von besonderer Schönheit hinterlassen hat. Er bat auch als der erste gezeigt, dass man Bacterien im Gewebe färben kann, er hat Färbungen für Fibrin, für elastische Fasern erdacht, die heute allgemein benutzt werden. In allgemein-pathologisehen Dingen von weitestem Blicke hat er uns zuerst gelehrt, dass diejenigen Processe im Nervensystem, bei denen Zellen und Fasern untergehen, die primären sind, dass das Zwischengewebe nie spontan wuchert, und nur die Stelle der durch Schwund der nervösen Elemente leer werdenden Räume einnimmt. Damit bat er für eine ganze Rejhe allgemeinpathologischer Untersuchungon auf dem uns beschäftigenden Gebiete den Ausgangspunkt geschaffen. Mitten in der Arbeit, nitten im Bestreben seine Glia- 
30. Wandervers. der Südwestdeutschen Neurologen u. Irrenärzte. 1023

methode zu verbessern, hat ihn eine Thrombose der Coronaria dahingerafft. Die Neurologie schuldet ihm für immer ein ehrendes Andenken!"

Die Anwesenden erheben sich um das Andenlien des Verstorbenen zu ehren, von ihren Plätzen.

Es folgen die Vorträge:

1. Herr Geh. Rath Bäumler-Freiburg stellt zwei Fälle vor, welche die Schwierigkeiten, die sich zuweilen bei der Beurtheilung von Nervenerscheinungen, die nach geringfügigen Verletzungen auftreten, ergeben.

Der 1. Fall betrifft einen 67jährigen sehr muskelkräftigen Mann, der v or $10 \mathrm{Jahren}$ durch das Zurückschnellen einer zusammengedrehten Weidenrute einen Schlag gegen die Ulnarseite der linken Hand erlitten hatte. Die ersten Erscheinungen, Schmerz und Schwellung, waren rasch vorübergegangen, da aber eine Gebrauchsunfühigkeit der beiden letzten Finger dieser Hand zurückblieb, wurde 3 Monate $n$ ach dem Unfall zum ersten Mal ein Arzt um Rath gefragt und erst weitere 5 Wochen später die Unfallsanzeige gemacht. Seitdem bezieht der Mann eine Rente, entsprechend einer Verminderung seiner Erwerbsunfähigkeit um $40 \%$.

Der z. Z. vorhandene Zustand ist zuerst in einer bezirksärztlichen Begutachtung vom October 1898 , also etwa $6 \frac{1}{2}$ Jahre nach dem Unfall, ganz so wie er jetzt sich darstellt, geschildert: die beiden letzten Finger krampfhaft eingeschlagen, lassen sich schwer, aber vollständig passiv strecken: zu activer Streckung ist der Kranke nicht zu bringen. Ausserdem besteht Herabsetzung der Empfindlichkeit bis zum Ellbogengelenk.

Mehrwöchentliche Beobachtung und Behandlung in der Klinik ergab, dass die erwähnte Haltung der beiden Finger mit grosser Willenskraft unterbalten wird. Beim Versuch, die Finger zu strecken, fühlt man deutlich die ruckweise zunehmende, mit grosser Willensanstrengung unterhaltene Contraction der Beugemuskeln, deren Ernährungszustand, ebenso wie der ihrer Antagonisten, ein ganz normaler ist. Ebenso das electrische Verbalten sämmtlicher Muskeln des Vorderarmes und der Hand.

Die Hartnäckigkeit, mit welcher der Kranke Streckungsversuche zu verhindern sucht, hat seit Beginn des Aufenthaltes in der Klinik allmählich noch zugenommen. Auch gab sich der Kranke keinerlei Mühe, durch eigene Vornahmen und Uebungen, zu welohen man ihn aufmunterte, die Finger in gestreckter Stellung zu erhalten.

Die Haltung der Finger im Schlaf zu beobachten, misslang, da der Kranke jedesmal dahei erwachte. Ihn zu chloroformiren erschien überflüssig angesichts des, abgesehen von der perversen Willensbeeinflussung, völlig normalen Verhaltens der betreffenden, wie sämmtlicher übrigen Muskeln des Armes und der Hand. Sehr bemerkenswerth ist ferner noch, worauf auch in dem. letzten bezirksärztlichen Gutachten aufmerksam gemacht wird, dass trotz dieser nach Angabe des Mannes seit 10, nach ärztlicher Bestätigung aber mindestens schon vor 8 Jahren bestehenden angeblich andauernden krampfbaften Eingesehlagenseins der beiden Finger, weder an der Hand- 
1024 30. Wandervers. der Südwestdeutschen Neurologen u. Irrenärzte.

fläche noch an den Fingern selbstirgendwelche Veränderungen, wie sie durch Druck oder Ernährungsstörungen schon nach sehr kurzer Zeit bei einer derartigen fixirten Stellang entstehen müssten (Eindrücke der Fingerspitzen oder der Nägel, Veränderungen an der Epidermis, Intertrigo in den Gelenkfalten) zu finden sind.

Neben dieser eigenthümlichen Haltung der Finger, über deren Natur wohl kein Zweifel bestehen kann, ist nun aber bei dem Kranken auch eine Sensibilitätsstörung nachweisbar, die ganz eigenthümlicb localisirt ist, and die in dieser Verbreitungsweise von einem der Anatomie Unkundigen nicht vorgetäuscht werden kann. Dieselbe betrifft nämlich genau den A usbreitungsbezirk des N. cutaneus medius vom Ellbogen abwärts und den N. uInaris in dessen der Sensibilität der Ulnarseite der Hand. wurzel and der Hand, sowie der 3 letzten Finger dienenden $\mathrm{Z}$ we ige mit völligem Freibleiben des motorischen Theiles des Ulnaris. Sämmtliche von diesem Nerven rersorgten Muskeln verbalien sich vollkommen normal. Längs des Verlaufs der N. cutan. medius, etwa an seiner Durchtrittsstelle durch die Fascie am Oberarm neben der Vena basilica oder weiter aufwärts, da wo der Nerv in der Tiefe neben dem Ulnaris liegt, ist nirgend wo durch Betastung etwas Abnormes nachweisbar.

Als eine hysterische"t lässt sich diese Anaesthesie nicht wohl ansehen, da bekanntlich bei Hysterie die Sensibilitätsstörungen nicht nach den Verbreitungsbezirken bestimmter sensibler Nerven sich localisiren. Auch ist dem Kranken die Localisation derselben nicht etwa durch häufge Untersuchungen suggerirt worden. In welcher Beziehung diese Leitungsunterbrechung in zwei Nerven zu dem vor 10 Jahren erlittenen geringfügigem Trauma steht, lässt sich mit Bestimmtheit um so weniger feststellen, als genauere Angaben über diese Störung aus den ersten Zeiten nach dem Unfall fehlen.

Im 2. Fall folgten bei einem sehr grossen und kräftigen Bauernburschen von 33 Jahren mebrere Monate nach einer oberflächlichen, aber mit ziemlichem Blutverlust einhergehenden, beim Holzhacken erlittenen Verletzung zwischen Zeigefinger und Daumen der rechten Fland (der Mann ist ein ${ }_{n}$ Linkser" und führte das Beil mit der linken Hand) eine zunehmende Erschwerung der Beweglichkeit der Hand des ganzen rechten Armes.

Einige Monate später - der Unfall hattè sich am 9. März 1903 ereignet, die Bewegungsstörung war zuerst im Herbst 1903 aufgetreten - am 1. Januar 1904 - trat ganz plötzlich, während der Kranlie mit Kameraden zusammensass und sang, ein Anfall auf, beginnend mit Schütteln des rechten Vorderarmes, woranf rasch völlige Bewusstlosigkeit folgte. Kein Zungenbiss. Solche Anfälle haben sich in ganz gleicher Weise, jedesmal mit Schütteln des Armes beginnend, in grösseren Zwischenräumen mehrfach wiederholt. Nie Kopfschuerzen, aber zuweilen etwas unsicheres schwindliches Gefühl im Kopf. Den Anfällen gehen keinerlei Vorboten voraus.

Die Untersuchung ergiebt einen mässigen Grad von Parese der ganzen rechten oberen Extremität und der Schultermuskulatur. 
30. Wandervers. der Südwestdeutschen Neurologen u. Irrenärzte. 1025

Die willkürlichen Bewegungen werden gleichmässig langsam, wie wenn eine Hemmung zu überwinden wäre, ausgeführt, ähnlich den Bewegungen eines Hemiplegikers nach Besserung der Motilität. Bei passiver Bewegung keine aufällige Rigidität. Keinerlei Sensibilitätsstörungen. Negativer ophthalmoskopischer Befund.

Der rechte Vorderarm und die Hand sind etwas cyanotisch und fühlen sich kühler an.

$1 \mathrm{~m}$ rechten Oberschenkel und zwar nur in diesem, nicht im Arm, bei längerem Stehen leichtes Zittern. Keine Störung des Ganges. Der Patellar- und Achillessehnenreflex, rechts etwas gesteigert, kein Babinski. Tiefe Reflexe am Oberarm nicht gesteigert. Im Gesicht keine Asymmetrie.

Die Erscheinungen sprechen für eine organische Erkrankung in den motorischen Stabkranzfasern, die von den Rindencentren für die rechte obere Extremität alislaufen, also in der linken Grosshirnhemisphäre (Subcorticaler Tumor?). Der Vortragende erwähnt einen von ihm beobachteten Fall, in welchem auf einen Arm beschränkt bleibende anfallsweise Paraesthesien und leichte Zuckungen Jahre lang der später deutlich gewordenen Entwicklung eines subcorticalen Tumors vorausgegangen waren.

Auffällig ist das Auftreten dieser Erscheinungen in einem gewissen zeitlichen Anschluss an die Verletzung mit Anfällen, die sebr an „Jacksonsche Epilepsie ${ }^{(1)}$ ) erinnern und die jedesmal in der verlotztgewesenen Extremität beginnen.

Discussion: Herr Fürstner möchte im zweiten Fall an die Möglichkeit einer pseudospastischen Parese denken und fragt, ob Tremor bestanden hätte.

Herr Bäumler verneint dies.

2. Harr Prof. Dr. Th. Axenfeld (Freiburg): Angeborene Bewegungsstörungen der Augen.

Vortragender stellt zunächst zwei Patienten vor, mit angeborener vollständiger Unbeweglichleit des linken Abducens, ohne secundäre Schielstellung beim Blick geradeaus und nach rechts, die Bewegung nach der Nase ist fast in normaler Breite ausführbar. Während bei erworbenen, dauernden Paralysen im Lauf der Zeit immer secundäres Schielen eintritt, kann also ein solches bei angeborener Unbeweglichkeit fehlen. Das liegt nicht, wie Kunn meint, daran, dass der Antagonist keine Neigung zur Contractur hätte, sondern daran, dass in solchen Fällen der Musculus abducens durch ein elastisches Band ersetzt ist, genügend straff, um das Schielen zu verhindern, genügend elastisch, uw den Blick nach innen nicht zu behindern. Vortragender hat dies anatomisch nachweisen können. Wo das den gelähmten

1) Nachtrag: Am Abend nach der Rückkebr von Baden.Baden in die Klinik hatte der Kranke einen Anfall, den ersten seit längerer Zeit. Ebenso an den beiden folgenden Abenden im Bett. Diese Anfälle konnten z. Th. ärztlich beobachtet und dabei festgestellt werden, dass völliger Bewusstseinsverlust und maximale Pupillen-Erweiterung und Starre vorhanden waren. Bei einem der Anfälle Zungenbiss und Schrei. 
1026 30. Wandervers. der Südwestdeutschen Neurologen u. Irrenärzte.

Muskel ersetzende Gewebe schlaffes Bindegewebe ist, tritt auch bei angeborener "Lähmung" secundäres Schielen auf. Wo der Ersatzstrang zwar sehr fest, aber nicht elastisch ist, bleibt das Secundärschielen aus, aber auch die Bewegung nach innen ist beschränkt; beim Versuch nach innen zu drehen, tritt, wenn der Antagonist nicht selbst paretisch ist, in manchen derartigen Fällen eine Retraction des Auges ein, an deren Zustandekommen auch eine Art von Retractor halb betheiligt sein kann.

Eine weitere sehr merkwürdige Möglichkeit, die von Hauck einmal anatomisch beschrieben warde und die Vortragender ebenfalls nachweisen konnte, ist; dass der dauernd und von Geburt an unbewegliche Muskel histologisch und seiner Stärkenach normal vorhanden sein kann, aber ohne eine Spur von activer Bewegung.

Es braucht also eine secundäre Muskeldegeneration bei constanter, angeborener Unbeweglichkeit nicht einzutreten, wieder ein Gegensatz zu dem Verhältniss bei später erworbenen dauernden Lähmungen der Augenmuskeln. Dass ein solcher inactiver Muskel auch für das Fehlen des Secundärschielens genügt, ist 7 weifelhaft, da in den beobachteten Fällen ein Strabismus bestand.

Vortragender demonstrirt schliesslich Bilder einer, angeborenen cyclischen Oculomotorins-Erkrankung". Er hat diese Bezeichnung gewählt, weil sich eine complete Paralyse aller Zweige des linken Oculomotorius vorfindet, die regelmässig alle $3-5$ Minuten durch einen Krampf unterbrochen wird: Das Oberlid wird maximal gehoben, der Bulbus rückt in die Mittellinie, die Pupille contrahirt sich und die Accommodation stellt sich auf den Nahepunkt. Nach circa einer halbe nMinute tritt wieder schlaffe Paralyse ein. Dies Spiel geht auch im Schlaf ununterbrochen weiter; die Patientin ist jetzt 12 Jahre alt und es ist anzunehmen, nachdem Vortragender 6 Jahre lang das Phänomen unverändert and bei sonst bestem Wohlbefinden der F. beobachtet hat, dass es so das ganze Leben weiter gehen wird. Der Fall erscheint deshalb so wichtig, weil in einem niemals activ beweglichen Gebiet diese rhythmischen Krämpfe erfolgen, also trotz der dauernden Lähmung Muskein, Nerv und Kern vollständig vorhanden und erregbar sind. Die sonst neurologisch bekannten Krämpfe in dauerud gelähmten Gebieten genen entweder allmälig in Contractur über (Facialis) oder sie machen schliesslich, wenn nicht Restitution eintritt, der Zerstörung Platz und hören auf. Am nächsten steht wohl die Hemiathetose nach cerebraler Kinderlähmung; doch wird von $H$. angegeben, dasis eine Spur von activer Beweglichkeit noch vorhanden sein müsse.

Es wird darauf zu achten sein, ob das ausnahmslos zutrifft. In der Literatur sind im Ganzen nur vier solcher Fälle bekannt. Als vorübergehende Erscheinung hat ausserdem Bechterew bei einer IIirnlues einmal einen rhythmischen Krampf des Levator beobachtet.

3. Herr Dr. Bayerthal-Worms: Zur Kenntniss der Meningocele spariamit Demonstrationen.

Auf das in neurologischer Beziehung vielseitiges Interesse darbietende Krankheitsbild der Meningocele spuria bat Vortr. bereits in einer früheren Arbeit die Aufmerksamkeit der Fachgenossen zu lenken versucht. (Deutsche 
med. W. 1889. No. 3.) Die in Rede stehende Anomalie gehört zu den grössten Seltenheiten auf dem Gebiete der Gebirnpathologie. Wohl in Folge dieses Umstandes hat die Meningocele spuria bisher in den Lebrbüchern der Gehirn- und Nervenkrankheiten noch keine Erwähnung gefunden. Yortr. stellt einen 24 jährigen Bauernburschen vor, der mit dieser Affection behaftet ist, und bespricht an der Hand dieses Falles kurz die Pathologie des "falsohen" Gebirnbruches. Was die Erscheinungen von Seiten des Gehiras anbelangt, so finden sich in dem demonstrirten Falle entsprechend dem Sitze der Meningocele über dem linken Parietale Paresen der-Fingermusculatur, Muskelatrophie und Wachsthumshemmung an Arm und Schulter der rechten Seite. Sensibilität an der rechten Hand herabgesetzt, psychologische Defecte fehlen. Von Interesse ist auch die Angabe des Patienten, wonach in den letzten zwei Jahren die rechte Hand in mehrwöchentlichen, unregelmässigen Zwischenräumen von einem tonischen Krampfe befallen wird, der mitunter mehrere Minuten anhält.

Für die. Diagnose sind die traumatische Entstehung im frühesten Kindesalter, Sitz und Form der Geschwult; ihr flüssiger Inhalt und ihre Communication mit derı. Schädelinnern maassgebend.

Die Prognose ist nicht durch die Geschwolst als solche, sondern durch die organischen Veränderungen des Gehirns bedingt, die noch im späteren Alter den Anstoss zur Entwicklung der Epilepsie geben können. Letztere wird bei genügend langer Beobachtungsdauer selten vermisst. In dem demonstrirten Falle ist diese bedenkliche Complication bis jetzt nicbt zu Tage getreten, wohl in Folge der geschützten Verhältnisse des Elternhauses, in denen Patient z. Zt. sich noch befindet, und seines hygienisch günstigen Berufes (Feldarbeit). Die genannten Folgen der mit der M. sp. verbundenen Gehirnerkrankungen scheinen in Ucbereinstimomung mit bekannten Erfahrungen auf dem Gebiete der Psychopathologie manchmal erst späterhin aufzutreten, wenn die Nothwendigkeit der eigenen Lebensführung, des Kampfes ums Dasein, die Sorge um Weib und Kind erhöbte Anforderungen an die physische Kraft des Individuums stellen.

Was die Therapie anbelangt, so dürfte der Standpunkt r. Bergmann's, der die "König'sche Operation behufs Heilung einer Meningocele sp. für ein Mittel zur Heilung der mit ihr verbundenen Epilepsie" hält, wohl kaum den Beifall des Neurologen finden. Zur Zeit, als der vorliegende Fall in die Beobachtung des Vortr. gelangte (1888), verfügte die chirurgische Technik noch nicht über diese Operation; möglicher Weise verdankt Patient zum Theil auch diesem Umstande, dass er bis heute von epileptischen Krämpfen verschont gebliehen ist. Vortr. erinnert in dieser Beziehung an die bekannten Erfahrungen Kocher's über den prophylaktischen Werth einer Schädellücke in Fällen traumatischer Epilepsie. Schliesslich weist Vortr. noch aus aktuellen Gründen. auf Hirndruckerscheinungen hin, die in der ersten Zeit der Beobachtung durch Compression der Geschwulst zu erzielen warew. Diese Repositionsversuche finden sich in den "Beiträgen zur klinischen Chirurgie", Bd. 7 , erwähnt. Sie sprechen entschieden für die Existenz eines Hirndruckes (gegen A da mkiew icz) und für den von Sänger neuerdings wieder begründeten mechanischen Ursprung der Stauungspapille. 
1028 30. Wandervers. der Südwestdeutschen Neurologen u. Irrenärzte.

4. Herr Prof. Fürstner (Strassburg): Ueber Hirntumoroperationen nnd dabei entstehende Hirnhornien.

F. hat im Jahre 1903 der Versammlung kurz über 4 Fälle berichtet, in denen die Diagnose auf Hirntumor gestellt, wo in dre: Fällen der Versuch gemacht wurde, die supponirte Neubildung operativ zo entfermen, während im vierten von vornherein nur ein palliativer Eingriff in Betracht kam. Zwei Kranke wurden im Mai 1902, einer im August desselben Jahres, der vierte im Januar 1903 operirt. Von ihnen leben noch zwei, der eine Kranke (im Mai operirt) starb nach einem Jahre, die zweite im Februar 1904.

F. berichtet zunächst über einen neuen Fall, der im Juni 1904 operirt wurde und 9 Monate später starb. 50jähr. Mann, die ersten Symptome im November 1903. Linksseitige Krämpfe, zunächst Bein, dann Arm, schliesslich Gesicht betheiligt mit Benommenheit. Anfangs grosses Intervall zwischen den Anfällen, dann zunehmende Frequenz, allmälig Parese links, am stärksten im Arm. Kopfschmerzen in der rechten Scheitelgegend, Stauungspapille, rechts mehr als links, rechts später auch Blutung. Progressir Abnabrae des Sehvermögens. Wegen hochgradiger Steigerung aller Symptone Operation; diagnosticirt wurde: corticaler Tumor in Centralwindung, das Hirn wird in zwei Etappen freigelegt über Armcentrum. Hirnsubstanz abgeplattet und stark vordringend. Tumor wurde nicht gefunden, bei Yalpation nirgends geringere Consistenz, mehrfache Punction ohne Erfolg. Verschluss des Defects mit Hautlappen, Heilung per primam. Zunächst Aufhören der Kopfschmerzen, Krämpfe, Rückbildung der Lähmung und Stauungspapille. Nach 14 Tagen kleiner pulsirender Prolaps, nach 3 Monaten Wiederkehr der Krämpfe, genau wie früher. Wachsthum der Hernie, schliesslich Längsdurchmesser $10 \mathrm{~cm}$, Breite 8, Tiefe $6 \mathrm{~cm}$. Schmerzhafte Anschwellung derselben bei Infällen. Von Neuem Verschlechterung, Abnahme der Intelligenz, beginnende Opticusatrophie, schliesslich fast Erblindung, spastische Parese links. Tod an Schluckpnenmonie. An der Hernie zwei Abthellungen, in vorderez massenhaft Flüssigheit, in hinterer liegen zwei Gyri, durch tjefen Sulcus getrennt, weisse Substanz partiell mit Tumor infiltrirt, an der Basis ganz mit Blutungen durchsetzte Tumorpartie. Grosse Neubildung, im Stabkranz nach links herüber sich ausbreitend, grosse Ganglien mit Tumorgewebe infiltritt. Letzteres zum Theil schleimig erweicht, Sitz unmittelbar unter der Rinde des Trepanationsgèbietes. F. hebt hervor, dass bei der Operation keine geringere Consistenz erkennbar war. Trotz der Trepanation musste der Tumor schnell weiter gewachsen sein; dafür sprach die Grösse der Hernien und zahlreiche osteoporotische Stellen im Felsenbein, auch in den Sinus longitudinalis Hirnhernien bereingewachsen. Erörterí werden die Wechselbeziehongen zwischen Hernienschwellung und Krämpfen, die Schmerzen bedingt durch periostitische Wrcherungen an der Trepanationsöfnung. Weiter wird tie Frage aufgeworfen, ob durch die Trepanations- und die Circulationsänderungen regressive Metamorphosen im Tumor begünstigt worden (Horsley), endlich die Entwickelung von Opticusatrophie nach Stavungspapille. Auch wenn der Tumor gefunden wäre, müsste er als inoperabel betrachtet werden. 
30. Wandervers. der Südwestdeutschen Neurologen u. Irrenärzte. 1029

Von den übrigen 4 Fällen sei Folgendes hervorgehoben: lm ersten wurde ein Tumor im Stirnhirn diagnosticirt (psychische Symptome, besonders Witzelsucht, Parese rechts, Fallen nach derselben Seite, Staungspapille). Tumor nicht gefunden. Zunächst Besserung, dann Wiederkehr aller Symptome, Erblindung, Tod darch T'uberculose. Bei der Obduction fanden sich 4 verkäste Tuberkel in der einen Kleinhirnhemispbäre. Auch hier zunächst grosse Hernie mit Flüssigkeit, die sich allmälig zurückbildete, so dass Galea dellenförmig über Defect lief. Hirnoberfläche hier erweicht. F. zieht analoge Fälle, namentlich einen von Albert publicirten, aus der Literatur heran.

Im II. Fall: Patientin hochgradig collabirt; da bis vor einem Jahre Ohreiterung bestand, war es fraglich, ob Abscess oder Tumor vorlag. Kopfschmerz, Stauungspapille sehr hochgradig, Fehlen des Fiebers, Schmerzlosigkeit des Processus mastoideus sprach für Tumor. Operation über dem Fuss der 3. Stirnund 1. Schläfenwindung. Nichts gefunden. Sehr grosse Hernie bildete sich erheblich zurück. Trotzdem der Augenspiegelbefund für beginnende Atrophie sprach, sehr erhebliche Besserung des Sehvermögens. Die sehr corpulent gewordene Kranke kann sich $21 / 4$.Jahr nach der Operation frei bewegen und etwas arbeiten. F. bespricht das Verhalten des Sehvermögens, das beiStauungspapille, beginnender Atrophie mit anatomischem Befunde nicht immer im Einklang steht, jedenfalls gelingt es, bei rechtzeitiger Minderung des Gehirndruckes die Erblindung hintanzuhalten. Im vorliegenden Falle war die Operation lebensrettend, auch jetzt andere Diagnose wie Tumor nicht möglich.

Im III. Fall: Operation über unterem Scheitelläppchen. Tumor nicht gefunden, nicht zn palpiren. Grosse Hernie, aus der sich einmal spontan, zweimal bei Punction eine grosse Menge gelber Flüssigkeit entleerte, die sich schnell wieder ersetzt. Auch hier Erhaltung des Sehvermögens. Rückgang der anderen Symptome. Tod fast zwei Jahre nach der Operation. Bei der Obduction fand sich ein grosser Substanztumor, subcortical bis in die andere Hemisphäre hineinreichend, in der Hernie Hirnsubstanz, mit der Galea verwachsen, in derselben ein grosse und kleinere Cyste.

Im IV. Fall bestand eine Rhinitis hypertrophica, dann Tumorsymptome. Localisation am vorderen Theile der Basis. Palliative Operation über dem Stirnhirn. Besserung aller Symptome trotz der ophthalmoskopisch festgestellten Atrophie, erhebliche Besserung des Sehvermögens. Auch hier Hernienbildung von wechselndem Umfang. Patientin steht noch in Beobachtung.

F. geht auf die Frage der Tumoroperation ein und spricht sich, da die Zahl der wirklich operablen Fälle sehr klein sei, zu Gunsten der Palliativoperation aus, die aber frühzeitig unternommen werden müsse, sobald die Stauungspapille erkennbar sei. Die rechtzeitige Minderung des Gehirndruckes sei zu erstreben und so das zu lange Bestehen der Stauungspapille mit ihren Consequenzen zu hindern. Die Palliativ-Operation komme in Betracht, wenn der Tumor nicht gefunden werde, wenn er sich als inoperabel erweise.

Schliesslich erörtert F. die Hernien, die den Gegenstand einer Dissertation des Herm Profé abgegeben. In Betracht komme die Entstehungszeit, der Inhalt der Hernie, die Bedeutung derselben für die Hirn- resp. Tumorsubstanz, 
1030 30. Wandervers, der Südwestdeutsclen Neurologen u. Irrenärzte.

vor Allem auch dịe Frage, welche Bedeutung die Hernie für den Gehirndruck haben kann.

5. Herr Dr. H. Curschmann (Tübingen): Zur Methodik der Muskel-und Gelenksensibilitäts bestimmung.

Da die bisherigen Methoden der Prüfung von Gelenks- und Muskelbewegungrgefühl (Goldscheider, Bernhard, Frenkel) eine quantitative Feststellung der Empfindungsschwelle, und somit in pathologischen Fällen einen zahlenmässigen Ausdruck des Herabsetzungsgrades der betreffenden Gefühlsqualität nioht ormöglichen, hat Vọtragender zur Prüfung beider Gefühlscomponenten die galvanomusculäre Methode gewählt.

1. Muskelcontractionsgefühl: Die Prüfung geschieht derart, dass bei möglichster Isolirung der betreffenden Extremität (Frenkel) zuerst die Minimalzuckung des Muskels und dann die Emplindungsschwelle des Contractionsgefühls desselben Muskels nach M. A. festgestellt wird. Die Differenzzahl zwischen beiden zeigt den Herabsetzungsgrad der Contractionssensibilität an. Unter normalen Verhältnissen fallen Minimalzuckung und Contractionsgefühl zusammen, resp. das letztere tritt schon um einige Zehntẹl M. A. früher auf, als das erstere. In pathologischen Fällen (Tabes, hemihyperästhetische cerebrale Hemiplegie, Syringomyelie, multiple Sclerose, periphere Lähmungen) mehr oder weniger hohe Grade ron Herabsetzung des Contractionsgefühls. Anwachsen der Differenzzahlen nach der Peripherie z. B. bei Tabes und cerebralen Hemiplegien. (Eine Curve zeigt Grad und Vertheilung bei hemihypästhetischer Hemiplegie.) Verwendbar ist die Methode auch zur Prüfung der hysterischen Tiefengefühlsstörung (Sensibilitätsverlust für den nbegrifflichen " Bewegungscomplex [Hellpach] oder auch für jeden einzelnen Muskel?). Auffallende Reducirung des Contractionsgefühls bei peripheren Lähmungen mit elektr. F. A. R. und constant geringe Herabsetzung desselben bei multipler Sclerose; regelmässige Verminderung des Contractionsgefühls bei $\mathrm{Sy}_{\mathrm{y}}$ ringomyelie im Bereich der dissociirten Empfindungslähmung.

2. Gelenksbewegungsgefühl: Nachdem durch galvanomusculäre Reizung die Minimalbewegung, $d_{\text {. }} i$. die kleinste sichtbare Bewegung in den betreffenden Gelenken festgestellt ist, ermittelt man die Gefühlsschwelle für die Gelenksbewegung. Beim Normalen fällt Minimalbewegung und Empfindungsschwelle zusammen.

Bei Tabes, hemihypästhetischer Hemiplegie, Syringomyelie, Myelitiden und Neuritiden beobachtet man mehr oder weniger grosse Differenzen zwischen Minimalbewegung und Empfindungsschwelle; Differenzzahl (in M. A. ausgedrückt) $=$ Grad der Herabsetzung der Empfindung. (An einer Curve Demonstration von Grad und Vertheilung der Störnng bei Tabes, bedentende Steigerung der Differenzzahlen nach der Peripherie der Extremität hin). Die Methode zeig't auch bei anataktischen Tabikern, bei denen nach gewöhnlicher Prüfung keine Gelenkstörungen zu erwarten sind, deutliche Störungen nach obigem Vertheilungsmodas an.

Schluss der Sitzung 1 Uhr Nachmittags. (Autoreferat.) 
30. Wandervers. der Südwestdeutschen Neurologen u. Irrenärzte. 1031

\section{Sitzung: 27. Mai 1905, Nachmittags 2 Uhr.}

Vorsitzender: Herr Hofrath Fürstner-Strassburg.

Es folgt zunächst der Vortrag von

6. Herrn Privatdocent Eduard Müller-Breslau: Ueber einige weniger bekannte Verlaufsformen der multiplen Sklerose.

Nach den Erfahrungen Strümpell's ist die multiple Sklerose bei der ländlichen Bevölkerung das häufigste grob-organische GehirnRückenmarksleiden; nur in der Grossstadt stelt sie hinter den syphilitischen bezw. metasyphilitischen Erlirankungen zurück. Die ,klassischent" Symptome (der eigentliche Nystagmus, das Skandiren und der echte Intentionstremor) sind nicht nur in ihrer Vereinigung selten, sondern in frühen. Krankheitsstadien auch einzeln keineswegs häufig. Andere und ebenfalls durchaus typische Zustandsbilder gestatten mit gleicher Sicherheit auch obne die Trias der "klassischen" Symptome eine richtige und dabei frühzeitigere Diagnose. Bei Berücksichtigung aller Verlaufsformen sind die Opticusaffectionen der multiplen Sklerose (namentlich in ihren charakteristischen Beziehangen zum Verhalten des Sehvermögens und zum Gesichtsfeld) das wichtigste und sicherste Symptom der multiplen Sklerose; sie finden sich in mindestens der Hälfte der einer Diagnose zugänglichen Fälle und sind von fast ausschlaggebender Bedeutung. Recht werthvoll ist das zuerst von Strümpell betonte Verhalten der Bauch deckenreflexe; sie fehlen meist schon im Beginn des Leidens. Bei jugendlichen und gesunden Personen mit normalen Bauchdecken sind sie jedoch eine sorgfältige, technisch richtige Prüfung rorausgesetzt - geradezu constant nachweisbar.

Vortragender bespricht dann das relativ häufige Einsetzen der Erkrankung mitflüchtigen, gelegentlich bis zu vorübergehender Erblindung sich steigernden Sehstörungen; sie sind meist nur das erste alarmirende, ausnahmsweise aber auch das einzige Frühsymptom. Ophthalmoskopisch findet man in solchen Fällen gewöhnlich temporale Abblassungen der Papillen mit kleinen centralen Sliotomen und neurologisch oft fehlende Bauchdeckenreflexe, das Babinslri'sche Zehenphänomen und ein leichtes Wackeln der Arme bei feineren Zielbewegungen. Sehr bäufig und bedeutsan ist weiterhin der Beginn des Leidens mit einer, allen Krankheitserscheinungen oft jahrelang vorauseilenden, abnormen Ermüdbarkeit; diese ist zum Unterschied von anämischen Zuständen und functionellen Nerven. leiden, deren Abgrenzung im frühesten Stadium der multiplen Sklerose nicht selten Schwierigkeiten macht, trotz annähernd gleicher äusserer Ansprüche an die Function gleichgebildeter Extremitäten ohne ersichtlichen Grund nur in dieser oder jener Extremität localisirt. Sehr selten ist die Entwicklung des Leidens mit heftigen neuralgischen Schmerzen; als anatomische Grundlage kommmen Herde in den Wurzeln der Gehirn- und Rückenmarksnerven in Betracht. 
1032 30. Wandervers. der Südwestdeutschen Neurologen u. Irrenärzte.

Zum Schluss weist der Vortragende auf die scheinbar paradoxe Thatsache hin, dass trotz der Vielgestaltigkeit der Erscheinungsweisen im Beginn der multiplen Sklerose die Grundzüge des Gesammtbildes meist auffällig. monotone sind. Die gemeinsamen, specifischen Merlkmale drängen sich auf, wenn man sich einerseits an die allgemeinen diagnostischen Gesichtspunkte hält und anderseits den zur Zeit bestehenden Symptomencomplex durch genaueste anamnestische Erhebungen ergänzt. Diese allgemein-diagnostischen Gesichtspunkte sind vor Allem das jugendliche Alter, das Fehlen wesentlicher äusserer Krankheitsursachen, das gewöbnlich schmerzfreie und gute Allgemeinbefinden der sonst meist durchaus gesunden und kräftigen Patienten, die sprongweise Entwicklung des Leidens unter Re- und Exaltationen. Bei der für die multiple Sklerose charakteristischen Flüchtigkeit und geringen Ausprägung diagnostisch wichtiger Einzelerscheinungen (z. B. AmLlyopien, Sprachstörungen, Doppelsehen, Blasenanomalien, Schwindelanfälle u. s. w.) muss man für die Frühdiagnose aus dem momentanen Zustandsbilde und den nur anamnestisch nachweisbaren Krankheitserscheinungen meist erst einen Symptomencomplex construiren. Dann gelingt es oft leicht, die fast stereotypen, quantitativ and qualitativ allerdings wechselnde Mischung von spinalen Stigmata einerseits, mit typischen Gehirn- und Augenstörungen anderseits nachzuweisen. (Erscheint ausführlich im "Neurologischen Centralblatt".)

Es erstattet sodann Herr Prof. Wollenberg (Tübingen) das Referat über: Die nosologische Stellung der Hypochondrie ${ }^{1}$ ).

Die ältere Literatur der Hypochondrie hat für heute nur noch historisches Interesse. Der Vortragende geht deshalb aufsie nur in soweit ein, als es zur Orientirung nothwendig erscheint: Nachdem zunächst die Anschauungen des Galenismus für die Auffassung derKrankheit maassgebend gewesen waren, sonderten sich mit der zunehmenden Erkenntniss der psychischen Genese der betreffenden Phänomene die Ansichten in zwei Gruppen: Die Einen hielten vach wie vor fest an der Bedeutung, die man von jeher den kranlihaft veränderten Hauptorganen der Bazchhöhle für die Entstehung der Hypochondrie beigelegt hatte, und liessen von diesen die psychischen. Störungen abhängig sein; die Anderen leiteten alles von der psychischen Störung ab. Dementsprechend unterschied man eine Hypochondrie cum und sine materia, eine Hypochondrie corporelle und mentale, sympathique und essentielle. -

In älterer "präneurasthenisober" Zeit bildete die Hypochondrie einen Sammelbegriff für die verschiedenen Neurosen. Insbesondere gab es keine Scheidung zwischen ihr und der Neurasthenie.

Der Vortragende geht kurz ein auf die Geschichte der letzteren seit Beard und weist, im Anschluss an Martius u. A., darauf hin, dass schon Jahrzehnte vor Beard die wesentlichen Erscheinungen, Verlaufsarten und Ausgänge der Neurasthenie bekannt gewesen seien, wenn auch unter anderen Namen (Spinalirritation und dergl.) - In Romberg's Lebrbuch der Nervenkranliheiten

1) Die ausführliche Veröffentlichung erfolgt demnächst in Centralblatt für Nervenheilkunde and Psychiatrie. 
30. Wandervers. der Südwestdentschen Neurologen u. Irrenärzte. 1033

finden wir dann die wesentlichsten Erscheinungen der Neurasthenie unter der Bezeichnung psychische Hyperästhesie oder unter dem gebräuchlicheren Namen "Hypochondrie" beschrieben und die Bedeutang des psychischen Momentes mit aller Schärfe betont.

Jedenfalls galt es bis in die 80 er Jahre des vorigen Jahrhunderts für eine unanfechtbare Thatsache, dass man es bei der Hypochondrie mit einer woblumgrenzten und selbständigen Krankheitsform zu thun habe. Hierin ist nun ein völliger Umschwung eingetreten, seitdem die Neurasthenie durch Beard Gemeingut der ärztlichen Welt geworden ist. Gegenwärtig bildet sie den weiteren Begriff, der die Hypochondrie in sich aufgenommen hat, und der Standpunkt der äberwiegenden Mehrheit der Fachgenossen lässt sich in dem Satze zusammenfassen: Hypochondrie ist nur eine Theilerscheinung, ein Cardinalsymptom der Neurasthenie.

Trotz der scheinbaren Prägnanz dieses Satzes ist hiermit aber eine hinreichend scharfe Umgrenzung der Hypochondrie nicht gegeben. Dies liegt an der Unbestimmtheit und Dehnbarkeit des Neurastheniebegrifes. Alle Versuche (Moebius, Kraepelin), die erworbene nerröse Erschöpfung von den hierher gehörigen angeborenen psychopathischen Zuständen zu trennen, haben zwar naturgemäss etwas Künstliches, trotzdem ist eine solche Trennung im Interesse der Klarheit nothwendig, und auch der Vortragende will in seinen Ausführungen unter Neurasthenie nur die durch chronisch erschöpfende Einflüsse erworbene Form verstanden wissen.

W. erörtert sodann unter Berücksichtigung der Literatur die Bedentung des Moments der psychischen Fyperästhesie für das Verständniss der Neurasthenie und die so häufige Entwicklung hypochondrischer Zustände, aus dieser letzteren heraus; dabei kommt er zu dem Schlusse, dass wir es hier mit keiner selbstständigen Krankheit, sondern nur mit einer besonderen Form der Neurasthenie zu thun haben, die nicht besser umschrieben werden könne, als dies in der bekannten Jolly-Hitzig'schen Definition geschehen ist.

Die weiteren Ausführungen beziehen sich auf die Fragen, ob es, abgesehen von dieser neurasthenischen Form, eine selbstständige echte Hypochondrie gebe, und welche nosologische Stellung dieser zuliomme. Eine kurze Uebersicht über die Bilder, unter denen uns die Hypochondrie klinisch entgegen. tritt, ergiebt zunächst, dass hypochondrische Episoden bei denverschiedensten psychischen Krankheitszuständen sehr häufig sind.

Vortragender erwähnt zum Beweise bierfür die entsprechenden Zustände bei Dementia paralytica und senilis, die hypochondrische Melan. cholie, die hypochondrischen Vorstellungen beim chronischen Alkoholismus, bei der Epilepsie und inshesondere bei den verschiedenen Formen der Dementia praecox, bei denen ibr symptomatischer Charakter wohl nicht selten verkannt werde.

An eine selbstständige Krankheit "Hypochondrie" lönnte man eher denken bei den nach Gemüthserschütterungen (Unfällen p. p.) entstehenden Fällen, die aber doch nur eine als traumatische Hypochondrie bekannte 
1034 30. Wandervers. der Südwestdeutschen Neurologen u. Irrenärzte.

Abart der traumatischen Neurosen („Schreckneurose" Kraepelin) darstellen. Hieran schliesst sich die Besprechung der auf dem Boden einer angeborenen psychopatischen Eigenart, zur Entwicklung kommenden Fälle, die theils der Hysterie, theils den Zwangszuständen, vor allem aber der "Nervosität" im engeren Sinne angehören. Hier finden eingehendero Berücksichtigung einmal die. sogenannten constitutionellen Verstimmten, sodann jene psychopathischen, die man als Fanatiker der Sorge um das ejgene körperliche Wohl bezeichnen und za den Pseudoquerulanten (Kraepelin, Aschaffenburg) in Parallele setzen kann.

Wenn alle bisher besprochenen Fälle ohne besondere Schwierigkeit zu bereits stehenden Krankheitsformen in nahe Beziebung gesetzt werden können, so bleibt endlich noch eine Gruppe von Fällen übrig, die sich durch die Entwicklung eines typischen hypohondrischen Wahns aber meist ohne eigentliche Systematisirung auszeichnen, und prognostisch günstig sind, jedenfalls nicht $z$ ur Verblödung führen, in didactischer Beziehung aber vielfach Schwierigkeiten machen. Ausgehend von einem Falle seiner Beobachtung spricht sich der Vortragende dahin aus, dass es sich auch hier nicht um selbstständige Krankheitsbilder handelt, sondern um jene constitutionellen Formen, die, wie $\mathrm{Kr}$ repelin sagt, eine sehr ausgesprochene Neigung haben, im Leben mehrfach, ja sogar sebr häufig wiederzukehren, d. h. zu den Formen, die wir im Allgemeinen als periodische zu bezeichnen pflegen, und deren ausgebildeten Typus das manisch-depressiveIrresein darstellt.

Dem entspricht anch der allseitig als ${ }_{n}^{\text {remittirend-exacerbirend }}{ }^{b}$ bezeichnete Verlaufscharakter dieser Fälle.

Das manisch-depressive Irresein besitzt eben eine viel grössere klinische Vielgestaltigleit, als man früber angenommen bat; insbesondere findet sich dabei lieineswegs selten eine recht hartnäckige Wahnbildung speciell hypochondrischen Inhalts, aber auoh ein ausgesprochener Verfolgungswahn liommt in den Depressionszuständen episodisch keineswegs selten vor und kann dann zu diagnostischen Schwierigkeiten führen. Eine innere Berechtigung, diese Fälle als eine selbstständige Formechter Hypochondrie abzugrenzen, besteht hiernach nicht; sie bilden eben eine der mannigfachsten Specialformen der auf degenerativer Basis entstehenden psychotischen Zustände.

Der Vortragende kommat hiernach hinsichtlich der Frage der Selbstständigkeit der Hypochondrie zu negativen Schlussfolgerungen. Wenn es auch mit Hülfe der Jolly-Hitzig'schen Definition leicht gelinge eine grössere Anzahl von Fällen zusammen zu hringen, so zeige sich doch bei näherer Betrachtung, dass diese sich sämmtlich in anderen bekannten Krankbeitsformen unterbringen lassen, und zwar komme hier - abgesehen von den hypochondrischen Episoden im Verlauf der anderen Geisteskrankheiten - vor Allem in Betracht: Die Neurasthenie in der vorhin gerebenen engeren Umgrenzung, sodann die Gruppe der constitutionellen Psychopathien. Die Hypochondrie ist eben nor ein psychopathologischer Zustand, eine krankhafte psychische Disposition besonderer Art, 
30. Wandervers. der Südwestdeutschen Neurologen u. Irrenärzte. 1035

also in letzter Linie ein Symptom. Immerhin kann dieses zuweilen eine so dominirende Stellung im Krankheitsbilde einnehmen, dass aus practischen Gründen für diese Fälle die Beibehaltung der Bezeichnang Hypochondrie, vielleicht auch der Unterabtbeilungen einer constitutionellen und accidentellen Form, gerechtfertigt erscheinen könnte. Hiermit soll aber die nosologische Selbstständigkeit der Hypochondrie bicht ausgesprochen werden.

(Autoreferat.)

\section{Discussion.}

Prof. Erb (Heidelberg) ist im Ganzen einverstanden mit den Schlussfolgerungen, welche der Herr Referent über die nosologische Stellung der Hypochondrie entwickelt hat; er erkennt jedoch in den klaren und interessanten Auseinandersetzungen desselben auf's Neue die grossen Schwierigkeiten, welche sich der Definition und schärferen Abgrenzung der klinischen Zustandsbilder und Krankheitsformen auf psychiatrischem Gebiet allenthalben - und nicht am wenigsten gerade bei der Hypochondrie - entgegenstellen.

Von bewerkenswerthem Interesse erscheint ibm der grosse Wechsel in den Anschauungen über denselben, welche sich in den letzten Jahrzehnten vollzog: vor 30 Jahren ging die damals noch neue "Neurasthenie" (Beard) gänzlich in der "Hypochondrie" auf; der ältere Westphal wies sie mit Entrüstung zurück und wollte gar nichts von ihr wissen, während E. schon frühzeitig die Richtigkeit der Beard'schen Aufstellungen anerkannte und in seiner grossen neurologischen Praxis bestätigt fand; heute ist man, wenigstens von manchen Seiten, nahe daran, die Hypochondrie in der Neurasthenie aufgehen zu lassen, sie nur als eine Theilerscheinung derselben, als eine $z$ u ihr gehörige Symptomengruppe anzusehen (abgesehen von einzelnen rein psychiatrischen Formen der Hypochondrie, der hypochondrischen Paranoia u. dergl., die ja auch der Herr Referent scharf herausgehoben hat).

E. kann darauf nicht näher eingehen, sondern gestattet sich nur einige Bemerkungen über die Auffassung der Neurasthenie, um Protest zu erbeben gegen die an manchen Stellen immer deutlicher hervortretende Anschauung, welche die Neurasthenie als eine Art der psychischen Erkrankung, als eine "Psychoneurose" betrachten möchten. Unter Hinweis auf die Formen der cardialen, vasomotorischen, gastrischen, cerebralen, spinalen Neurasthenien u. a. glaubt er aussprechen zu dürfen, dass diese Formen doch ganz gewiss keine "psychische Erkrankung" darstellen. Psychische Symptome und Störungen; und speciell hypochondrische Erscheinungen fehlen ja dabei in vielen Fällen durchaus nicht; besonders wären dahin die zahlreichen Angstzustände (neurasthenische "Phobien") zu nehmen und unter diese kann sich wohl, wie ja bereits geschehen, auch die Hypochondrie als eine besondere Form, als „Nosophobie" eingerechnet werden; aber das nimmt der Neurasthenie nichts von ihrer Selbstständigkeit.

Dasselbe Symptomenbild kommt doch auch bei zahllosen anderen Erkrankungen (des Darmes, des Herzens, der Nieren, des Rückenmarks etc.) vor und erlangt nur in einer gewissen Reihe von Fällen eine grössere oder selbst

Archiv f. Psychiatrie. Bd. 40. Heft 3. 
1036 30. Wandervers. der Südwestdeutschen Neurologen u. Irrenärate.

vollständige Selbstständigkeit, und ist dann als eine rein psychische Erkrankung aufzufassen.

Jedenfalls erscheint es E. nicht gerechtfertigt, die Neurasthenie, in deren Symptomenbild neben anderen psychisehen Anomalien auch die Hypochondrie eine hervorragende Rolle spielen kann, in ihrer jetzt allgemein anerkannten Selbstständigkeit fallen zu lassen.

(Autoreferat.)

Herr Edinger (Frankfurt): Wir wissen, dass durch Herde im Gehirn Schmerzen nach aussen projicirt werden können; das ist klinisch und anatomisch nachgewiesen. Da dürfte es sich wohl verlohnen, in Zukunft bei der Beobachtung organischer Herdläsionen darauf zu achten, ob nicht in das eine oder andere Organ Schmerzen projicirt werden. Es ist leicht möglich, dass manche Formen gewissermaassen localisirter Hyponchondrie auf Herde zurückgutährt werden können; mit solchem Nachweis wäre gewiss viel gewonnen.

Herr Fürstner (Strassburg) glaubt nicht, dass es viele Leute giebt, welche die Neurasthenie grundsätzlich als Psychose ansehen; wohl aber ist er auf Grund vielseitiger Erfahrung zu der Ueberzeugung gekommen, dass die psychischen Symptome, die bei der Neurasthenie vorkommen, foft übersehen und noch bäufiger in ihrer Bedentung überschätzt werden; die richtige Bewerthung der psychischen Symptome sei wichlig, sowohl für die prognostische Beurtheilung als für die Therapie. Bezüglich der Hypochondrie betont Fürstner, dass es nothwendig sei, von der symptomatischen hypochondrischen Verstimmung scharf zu unterscheiden die angeborene, constitutionelle, hypochondrische Verstimmung, die im späteren Leben zu einer hypochondrisehen Parunoia sich ausgestalte; für diese Form der Erkrankung sei unbedingt der Begriff Hypochondrie als selbsständige Psychose aufrecht za erhalten.

Herr Prof. Erb erkennt die Berechtigung der von dem Vorredner gemachten Ausführungen vollkommen an; er glaubt ebenfalls, dass in der Praxis die bei der Neurasthenie auftretenden psychischen Störungen vielfach ïbersehen und nicht genügend gewürdigt werden, vielmehr, dass ausserordentlich häufig unzweifelhaft primäre psychische Erkrankungen irthümlich oder absichtlich unter den unverfänglichen Sammelbegriff der "Neurasthenie" untergebracht werden. Er wollte nur darauf hinweisen, dass die Neurasthenie eine wohlbegründete selbstständige Krankheitsform ist, bei welcher keineswegs immer psychopathische Symptome vorhanden sein müssen und welche man jedenfalls nicht einfach unter die "Psychoneurosen" einreihen darf.

8. Herr R. Friedlaender (Wiesbaden): Ueber Störung der Gelenksensibilität bei Tabes dorsalis.

Vortragender berichtet übor Untersuchung der Bewegungsempfindung an den unteren Extremitäten bei 27 ataktischen Tabikern. Die primäre Störung betrifft die Lageempfindung, die Bewegungsempfindung als solche wird erst später beinträchtigt. Die ersten Anomalien zeigon sich stets in den Zehengelenken; je schwerer der Grad der Ataxie, desto mehr Gelenke proximalwärts sind an der Störung betheiligt: bei leichter Ataxie bleiben Knie- und Hüftgelenke in der Mehrzabl der Fälle frei, bei schwerer Ataxie ist die Bewegungsempfindung in der Regel in sämmtlichen Gelenken herabgesetzt. Sind mehrere 
30. Wandervers, der Südwestdentschen Neurologen u. Irrenärzte. 1037

Gelenke dieser Extremität afficirt, dann findet sich - besonders in leichteren. Fällen - fast immer proximalwärts allmälige Abnabme der. Störung der Bewegungsempfindung von einem Gelenk zum anderen. Ist ein Gelenk normal, dann ist es auch in der Regel das näcbst böhere. Der Grad der Störung der Bewegungsempfiodung entspricht durchaus nicht immer dem Grade der Ataxie, wern auch relativ an dem stärker ataktisehen Bein gegenüber dem weniger ataktischen meist intensivere Störungen zu constatiren sind. Das Missverhältniss zwischen Grad der Ataxie and Störung der Bewegungsempfindung ist in einzelnen Făllen besonders auffallend in Bezug auf statistische Aufgaben. Zụ Erklärung dieser Incongruenz dienen in erster Reihe individuelle Unterschiede bezüglich der Reaction auf sensible Reize, sowie der motorischen Einübung und Geschicklichkeit, dann das Verhalten der Hantsensibilität und besonders der Muskeltonus. Nicht nur die Störungen der bewussten Sensibilität, sondern auch der Ausfall unbewusster, subcorticaler sensibler Merkmale scheint für die Entstehung der Ataxie von Bedeutung zu sein.

9. Herr Dr. Siegmund Auerbach (Frankfurt a. M.): Ueber einen mit Erfolgexstirpirten Tumor des Cervicalmarkes.

Vortragender demonstrirt das durch die Operation gewonnene Präparat (6 cm langes Fibrosarkom). Die Patientin selbst ist bereits von Dr. Brodnitz auf dem diesjährigen Chirurgen-Congress kurz vorgestellt worden. Der Tumor, der von den Arachnoidealséheiden der hinteren rechtsseitigen Wurzeln des mittleren Cervicalmarkes ausgegangen war, comprimirte das ganze Halsmark von rechts, hinten und links her vom Foramen magnum bis zum 1. Dorsalsegment. Er ist der grösste bisher beobachtete extramedulläre, intradurale des Rückenmarkes überhaupt, sicherlich aber des Cervicalabschnittes; jedenfalls ist ein so ausgedehnter bisher noch nicht exstirpirt worden.

Der klinische Veriauf wird kurz skizzirt. Die Differentialdiagnose gegenüber der Pacbymeningitis hypertrophica, der Syringomyelie, der Lues spinalis wird ausführlich erörtert. Alsdann wird der muthmaassliche Sitz, ob vertebral, intramedullär oder meningeal eingohend besprochen, obenso die Niveandia. gnose. Weiterbin wird das erhebliche Ueberwiegen der motorischen und muskeltrophischen Symptome über sensiblen Ausfallserscheinungen, die nur ganz vorübergehend und in geringer Ausdehnung nachzuweisen waren, durch die speciellen topographischen Verhältnisse zwischen Tumor und Medulla erklärt. Hierauf wird kurz der postoperative Vellauf in neurologischer Beziehung besprochen und bervorgehoben, dass bereits jetat (6 Monate nach der Operation) eine fast röllige Heilung eingetreten ist. Nur die oculopupillären Symptome, der sogenannte Horner'sche Symptomencomplex, sind nicht zurückgegangen. A. sucht diese Thatsache zu erklären und betont, dass sein Erlilärungsversuch mit den experimentellen Ergebnissen Langley's gut zu vereinbaren ist.

Die Lehren, die er aus seiner Beobachtung gezogen hat, fasst Vortragender in folgenden Sätzen zusammen:

Bei Erscheinnngen von Räckenmarkscompression denke man stets awch, namentlich dann, wenn eine Geschwulst im Niveau des Halsmarkes in Frage kommt, an die Möglichkeit, dass die dem Tumar gegenüberliegende Seite der 
1038 30. Wandervers. der Südwestdeutschen Neurologen u. Irrenărzte.

Medulla bedeutend mehr (oder auch allein) geschädigt sein kann, als die ihm direct anliegende.

Die Regel, dass intradurale Tumoren meistens klein, extradurale am häufigsten gross sind, hat ihre Ausnahmen.

Die durch Druck auf das Centrum ciliospinale erzeugten oculopupillären Symptome können trotz des Rückganges aller übrigen Lähmungserscheinungen bestehen bleiben.

Die Differentialdiagnose zwischen Wirbelcaries und Tumor des Cervicalmarkes ist in manchen Fällen trotz eingehendster Berücksichtigung aller bekannten diagnostischen Momente nicht mit absoluter Sicherheit zu stellen. Insbesondere lasse man sich durch das Ueberwiegen der motorischen und muskeltrophischen Störungen über dic sensiblen nicht von der Annahme einer Geschwulst abhalten. Sind die Zweifel nicht zu zerstreuen, so soll man dem Kranken unter offener Erklärung der Sachlage, der Aussichtslosigkeit seines Leidens einerseits, der nach neueren Beobachtungen nicht allzu grossen Gefahren der Operation als einziger Rettungschance andererseits, oher zu dem chirurgischen Eingriff rathen. (Autoreferat.)

Die Arbeit wird ausführlich in den „Mittheilungen aus den Grenzgebieten der Medicin und Chirurgie" veröffentlicht werden.

10. Herr Dr. Spielmeyer (Freiburg i. B.): Ueber familiäre amaurotiscbe Idiotien.

Unter den Fällen familiärer Idiotie, die sich mit Amaurose compliciren,

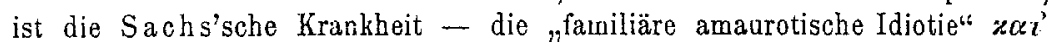
$\varepsilon \xi \circ \not \dot{\eta} \nu$ - gut abgegrenzt. Durch die constante Verbindung ihrer beiden Hauptsymptome, der unter allgemeinen Lähmungserscheinungen rasch fortschreitenden. Verblödung und der charakteristischen Maculaveränderungen, ist sie ausgezeichnet vor jenen Fällen, in denen idiotische Geschwister gleichzeitig auch amaurotisch sind und in denen die Blindheit lediglich die Bedeutung eines Begleitsymptomes bat (manche Mikroencephalien mit schweren Entwicklungshemmungen am Auge, vereinzelte familiär auftretende Verblödungsprocesse verbunden mit Chorioiditis oder Sehnervenatrophie etc.).

In die Reihe dieser letzten Erkrankungen, vielleicht aber auch zu einer selbstständigen Form familiärer amaurotischer Verblödung gebört eine eigene Beobachtung des Vortragenden: eine erworbene Idiotie, die zusammen mit Erblindung bei vier Geschwistern in ganz gleicher Weise verlief. Nur das älteste Kind dieser Familie blieb gesund. Nach dessen Geburt luetische Infection (?) des Vaters. Beginn der Erkrankung bei allen 4 Kindern zur Zeit der zweiten Dentition. Stets die gleiche Trias der Symptome: epileptische Anfälle, rasche Verblödung und schnell fortschreitende Erblindung (Retinitis pigmentosa). Einige nebensächliche Differenzen im Krankheitsbilde bei dem jüngsten Kinde (epileptische Anfälle nur im Beginne des Processes, Erregungszustände, transcortical aphasische Störungen) - keine Erscheinungen, die auf eine herdförmige oder systemartige centrale Läsion oder auf eine infantile Paralyse zu beziehen gewesen wären. 
30. Wandervers. der Südwestdeutschen Neurologen u. Irrenärzte. 1039

Die in einem dieser Fälle vorgenommene anatomische Untersuchung ergab eine diffuse Erkrankung des centralen Nervensystems, die am ausgesprochensten in der Rinde ist und deren wesentlichstes Characteristicum eine eigenartige Zellerkrankung ist (Ablagerung eines körnigen, oft pigmenthaltigen Stoffes, Aufblähung des Zellleibes etc). Auf eine Besprechung des anatomiseben Befundes wird der Kürze der Zeit wegen verzichtet; Vortr. legt einige Abbildungen vor, die die wesentlichsten Veränderungen der $\mathrm{Nissl-}$ Schollen, der Fibrillen, der Gliazellen und Gliafasern illustriren sollen. Vortragender weist nur kurz darauf hin, dass dem eigenartigen kinischen Bilde ein scharf gekennzeichnetes anatomisches Substrat entspricht, das von den bisher bekannten Rindenbildern abweicht.

In einer ausführlichen Publication sollen die klinische Stellung dieser Fälle und vor Allem die pathologischen Veränderungen besprochen werden.

(Eigenbericht.)

Herr Privatdocent Dr. Stock (Freiburg i. B.): Retinitis pigmentosa bei den von Spielmeyer erwähnten Fällen.

Vortragendor hat ron den 4 Kindern 3 mit dem Augenspiegel untersuchen können. Bei den zwei ältesten fand sich das typische Bild der Retinitis pigmentosa: Verengerte Retinalgefässe, Pigmenteinwanderung in die Retina in den sogenannten Knochenkörperchenfiguren. Bei dem dritten Kinde besteht Amaurose, der Augenhintergrund ist nicht pathologisch verändert. Es handelt sich also in diesem Fall um eine sogenannte Retinitis pigmentosa sine pigmento.

Pathologisch-anatomisch wurde ein Bulbuspaar untersucht. Dabei konnte festgestellt werden, dass in der Retina die Stäbchen- und Zapfenschicht fehlt, ebenso von der äusseren Köruerschicht der grösste Theil zu Grunde gegangen ist, während die Nervenfaserschicht und innere Körnerschicht noch wohl erhalten sind. Die Retinalgefässe sind sehr erheblich verändert, zum Theil obliterirt, zum Theil ist ihr Lumen durch endarteriitische Processe sehr stark verengert. In die Scheiden der Gefässe ist Pigment eingewandert, welches zweifellos aus den Pigmentepithelien der Retina stammt.

Einen Unterschied gegen die früher beschriebenen Fälle (Gonin, Wagen $\operatorname{mann}$ ) ist insofern vorhanden, als die Veränderungen in der Choroidea nur sehr gering sind. Die Choriocapillaris ist jedenfalls auf weite Strecken erhalten und ganz besonders an Stellen vorhanden, über welchen schwere Degenerationen in der Retina zu seben sind.

Als einen specifisch syphilitischen Process kann man nach der Ansicht des Vortragenden diese Retinaldegeneration nicht auffassen, wohl aber ist es erlaubt anzunehmen, dass eine allgemeine Ernährungsstörung in Folge von angeborener Syphilis einmal eine solche Netzhautdegeneration versursachen kann.

11. Herr Prof. Dr. Karl Schaffer (Budapest): Zur Pathobistologie der Sachs'schen amaurotischen Idiotie.

Vortragender resumirt vor Allem seine in den No. 9 und 10 des Neurolog. Zentralb. a. c. veröffentlichten Untersuchungsergebnisse bezüglich der Pathohistologie der Sachs'schen amaurotischen Idiotie, ron welchen er besonders 
1040 30. Wandervers. der Südwestdeutschen Neurologen u. Irrenärate.

die Schwellung der Nervenzelle als hervorstechendstes Moment heraushebt. Diese Schwellung kann sich theils in einer, auf den ganzen Zellkörper erstreckenden, also to tal en Schwellungzeigen, theils aber als locale Autblähung exscheinen. Beachtenswerth erscbeint es, dass die Dendriten zumeist unverändert sind, wogegen der Zellkörper bereits tiefgehende Veränderongen aufweist, Blutgefässe überall normal; Entzündung nirgends zu sehen.

Vortragender schildert nun seine neueren Ergebnisse, welche er am Centralnervensystem seines neuesten (7.) Falles von Sachs'scher Idiotie gewann. Diese stimmen wesentlich mit seinen oben geschilderten Resultaten überein; doch gestatteten vollkommene Bielscbowsky-Imprägnationen, welche mit Zeiss' Aprochomat-Immersion $2 \mathrm{~mm}$ and Compensationsocular 18 analysirt wurden, einen tieferen Einblick, nicht nur in die pathohistologischen Vorgänge, sondern auch in die Normalstructur der Nervenzelle, letzeres umsomehr, da mit der Fibrillenmethode noch normale oder naheza normale Nervenzellen, wenn auch vereinzelt, sich vorfanden. So liess sich vor Allem nachweisen, dass die Nervenzellen ein distinktes Golginetz besitzen, welches different gebaut scin kann. Man sieht neben der polygonalen Maschenstructur Stellen an demselben Golginetz, welche parallel-streifig, quasi fibrilläl gebaut erscheinen: die genaue Analyse ergab aber, dass diese Fibrillen mit schrägverlaufenden anastomotischen Fäden - wodurch oblonge Maschen entstehen untereinander verbunden sind. Dies durch das Golginetz reprïsentirte Aussenreticulum' der Nervenzelle bängt continuirlich mit einem, aus lockeren Maschen bestehenden Innenreticulum zusammen, welches den Zellleib sowie die protoplasmatischen Fortsätze durchsetzt. Somit ist die Nervenzelle reticulär gebaut; das, was hisher Fibrillen genannt wurde, ist nichts anderes, als stärkere longitudinale Züge des Golginetzes. Der Nervenzellkörper besteht nach der Auffassung des Vortragenden ausser dem bislang ungeformten Plasma aus dem s. g. Neuroreticulum, welches aus einem Aussenreticulum oder Golginetz und aus einem Innenreticulum besteht; ersteres baut sich im Allgemeinen aus derberen, kräftigeren Trabekeln auf, während letzteres aus viel feineren Fäden gebildet wird. Letztere sind um den Kern herum in dichteren Masclien zu sehen.

Bei der Sachs'schen Idiotie zeigt sich zuerst eine Schwellung des Innenreticnlums, wodurch die Lücken kreisrund, aufgebläht werden; zugleich erscheinen die Knotenpunite durch Auftreibung vergrössert. Später verschwinden die verbindenden Trabekel der Knotenpunkte des Innenreticulums, so dass nur die Knotenpunkte als sternförmige gezackte Körner. zurückbleiben. Schliesslich ist der Zellkörper mit Granulis hesät und da ist von einer Netzstructur nichts mehr zu schen. Zu diesem terminalen Stadium der Nervenzellerkrankung ist der Zellkern auch krankhaft verändert; er tingirt sich tief, wird rissig, schwammig und gezackt.

Das Golginetz oder Aussenreticulum ist auch in Fällen ron rorgeschrittener Nervenerkranlinng erhalten. Der Process beginnt eber central und schreitet successive gegen die Peripherie.

Die Nisslbilder weisen durchweg eine hochgradige Chromolyse auf 
30. Wandervers. der Südwestdeutschen Neurologen u. Irrenärzte. 1041

und es ist interessant and wichtig, dass die Nisslfärbung auf hochgradigere Erkrankungen hinweist, wie die Fibrillenfärbung. Hieraus dürfte gefolgert werden, dass die Nisslsubstanz viel empfindlicher ist, viel leichter zorfällt wie das Neuroreticulum. Auch wäre hervorzuheben, dass mit der Nisslfärbung reticulirte Structuren sichtbar sind, welche dem Cayal'schen Spongioplasma entsprechen.

Die Markscheidenbilder zeigen eine hochgradige Armuth an Mark. fasern (hauptsächlich im Frontal- und Temporallappen). Ausserdem ist an Frontalschnitten durch ganze Hemisphären in der gelben Rinde ein graulicher Streifen bereits mikroskopisch sichtbar, welcher, mit der Oberfläche parallel verlaufend, etwa einem Baillarger entsprechend, in der Rinde sämmtlicher Lappen verläuft, die Lage und Gestalt der Rindenzelle nachahmend. Es sind dies krankbaft veränderte Nervenzellen, wie dies der Vergleich mit Fibrillenpräparaten zeigt. Somit haben wir in den Markscheidenpräparaten ein bequemes Mittel, die Ausdehnung des Processes zu verfolgen; diese ist die denkbar grösste. Die ganze Rinde, der Hirnstanm, das Rückenmark ist ergriffen. Bezüglich des Opticus erwähnt Vortragender, dass derselbe mit der Markscheidenfärbnng sowie auch mit der Fibrilieniärbung als ganz normal sich erwies; die Erblindurg ist cortical bedingt, denn die Calcarinarinde weist die höchstgradige Zellerkrankung auf:

Schliesslich ist zu bemerken, dass an die Stelle der zu Grunde gegangenen Nervenzellen die Glia rückt. Diese erscheint theils in der Form von den bekannten Spinnenzellen, theils als kugelförmige, hypertrophische Zellen, welche, in Nester gruppirt, die Stelle der ehemaligen Nervenzellen einnehmen; letztere Gliazellen zeigen eine Tendenz zur regressiven Metamorphose.

(Autoreferat.)

12. Herr Professor Dr. Weintraud (Wiesbaden) berichtet über vier Fälle, bei denen er, zum Theil nehrmals, nach der zuerst von A. Kocher angegebenen, dann neuerdings von A. Neisser empfohlenen Methode, Punctionen des Gehirns zu diagnostischen und therapeutischen Zwecken ausgeführt hat.

Dabei wird nach localer Anästhesie der Schadelhaut durch Andrücken eines elektrisch angetriebenen Drillbohrers die Galea und der Knochen durchbohrt und die Punctionsnadel direct durch die gewonnene kleine Oeffinung eingeführt.

In dem ersten Fall gaben vorübergehende Erscheinungen von sensorischer Aphasie einen Anhaltspunkt, bei der halbseitig gelälmten Kranken den linken Schläfenlappen zu punctiren, als sie in tiefes Coma verfallen war. Bei der Punction entleerte sich eine grosse Menge klarer, goldgelber Cystenflüssigkeit. Die Kranke erwachte aus ihrem Coma. Die Besserung war aber von kurzer Dauer und auch durch eine erneute Punction lionnte das Eride nicht abgewendet werden.

Bei der Autopsie fand sich ein grosser Tumor, der den ganzen linken Schläfenlappen ausfiullte und in seinen vorderen Partien gelatinös und cystisch verändert war.

In einem zweiten Fall wiesen schwere Hirndrucksymptome (Erbrechen, 
1042 30. Wandervers. der Südwestdeutschen Neurologen u. Irrenärzte.

Kopfschmerz, Pulsverlangsamung, Stauungspapille) anf das Vorhandensein eines Hirntumors hin, olne dass für seine Localisation ein Anhaltspunlit gegeben war.

Die Wahrscheinlichkeitsdiagnose lautete auf Solitärtuberkel mit Hydrocephalus internus. Die jetzt im Ganzen dreimal in etwa vierwöchentlichen Intervallen ausgeführte Punction der Seitenventrikel des Gehirns brachte durch die Entleerung von je $20-40 \mathrm{~cm}$ Cerebrospinalflüssigkeit ganz ausserordentliche Besserung in dem Befinden der Kranken, deren Zustand vorher durch die mit keinem Mittel zu beseitigenden Kopfschmerzen und schliesslich durch eintretende Somnolenz und Schwierigleit der Nahrungszufuhr lebensgefährlich geworden war.

Interessant war das vollkommen negative Ergebniss der Ventrikelpunction bei einem jungen Mädchen, das nach einem Krampfanfall in das Krankenhaus eingeliefert, daselbst zuerst Symptome einer Psychose, dann Benommenheit und starke Pulsverlangsamung und Stauungspapille aufwies. Es entleerte sich kein Tropfen Flüssigkeit aus der Gehirnsubstanz. Die Kranke starb wenige Tage nach der Punction im Coma und die Autopsie ergab keine anatomische Erklärang für die Hirndruckerseheinungen, vor allem keinen Tumor und auch keinen ausgesprochenen Hydrocephalus internus. Lie Abplattungen der Hirnwindungen und die Trockenheit der Hirnsubstanz, zusammen mit dem negativen Ausfall der Ventrikelpunction lassen den Fall als einen solchen von Hirnschwellung (Reichardt) auffassen.

Die Aspiration von Blut aus dem Plexus des Ventrikels in einem Falle von tuberculöser Meningitis beeinflusste den letalen Verlauf der Krankheit nicht. Eine Blutung in den Ventrikel und in die Hirnsubstanz war dabei, wie sich bei der Autopsie controliren liess, nicht erfolgt.

Bei Hirntumoren empfiehlt es sich, nicht zu warten, bis sichere Herdsymptome die Localisation und damit den Eingriff ermöglichen, sondern durch frühzeitige event. mehrfache Punction der sonst so häufig eintretenden Erblindung vorzubengen.

13. Herr Dr. Oscar Kohnstamm (Königstein i. Taunus): Die Kerne des prädorsalen Längsbündels und des Trigeminus. Ein Beitrag zur topischen Diagnostik der Oblongata.

Es ist bekannt, dass die Fasern der fontainenartigen Haubenkreuzung in dem der Ursprungsseite gekreuzten prädorsalen Längsbündel caudalwärts ziehen, um in der Oblongata und im Rückenmarke za endigen. Als Ursprung hatte ich einen als Nucleus intratrigeminalis tecti bezeichneten Kem angegeben, dessen grosse motorische Zellen in den mesencephalen Trigeminuskern des vorderen Vierhügels eingestreut sind (Monatsschr. f. Psychiatrie und Neurologie 1900. Neurolog. Centralblatt 1903). Das im oberen Cervicalmark hemisecirte Kaninchen, bei dem offenbar nur wenige Fasern bis ins Halsmark hinabsteigen (Marohi Methode), zeigte bei Nissl-Behandlung beiderseits degenerirte Zellen des Nuclens intratrigeminalis. Die Erklärung für die Degeneration auch auf der Operationsseite sehe ich jetzt darin, dass der Halbseitenschnitt durch das obere Halsmark das prädorsale Längsbündel der nicht durch- 
schnittenen Seite mitverletat hat, welches ja der vorderen Längsspalte dicht anliegt.

Gegen meine Auffassung des Nucleus intratrigeminalis hat M. Lewandowsky in einer inhaltreichen und werthvollen Arbeit sehr temperamentrollen Einspruch erhoben und den Kern als Ursprung des "Tractus Probsti“ angesprochen, jener räthselhaften Fortsetzung der mesencephalen Trigeminuswurzel bis hinab in die Gegend ventral vom dorsalen Vaguskern (M. Le wandowsky, Unters. über die Leitungsb. des Truncus cerebri. S. 113 und 119. Jena 1904),

Durch die Liebenswürdigkeit des Herrn Dr. Bickel hatte ich Gelegenheit, in der ihm unterstehenden experimentellen Abtheilung des Berliner pathologischen Institutes meinen Versuch am Hund zu wiederholen and mit der $\mathrm{Nis}$ i-Methode festzustellen, dass bei diesem Thier in viel ausgesprochenerer Weise als beim Kaninchen nach Hemisection des Halsmarkes der Nucleus intratrigeminalis der Gegenseite degenerirt und demnach als Ursprung des prädorsalen Längsbündels angesprochen werden muss (Demonstration). Wallenberg ist durch Untersuchnng des Taubenhirns ebenfalls zu der Schlussfolgerung geführt worden, dass tiefes Mark und cerebrale Quintuswurzel annähernd gleichen Ursprung besitzen. (Anat. Anz. Bd. XXV.)

Die weisse Schicht, in welcher die Axone des Nucleus intratrigeminalis zur Fontänenkreuzung ziehen, bildet die mediale Begrenzung des tiefen Markes und wird zweckmässig als Stratum marginale des centralen Höhlengraus bezeichnet. Die dorsal und lateraI rom tiefen Mark gelegene grave Substanz des vorderen Vierhügels wird durch die weisse Sehnervenschicht in eine äussere und innere graue Zone getheilt. Die innere graue Zone stellt Golgi-Präparaten zufolge (vergl. Kölliker) die Verbindung her zwischen der Sehnervenschicht und den Fasern der Fontänenkreuzung. Da von dieser, beziehungsweise dem prädorsalen Längsbündel Collateralen zu den Augenmuskelkernen gehen, so stehen wir hier an der Stelle der engsten Verbindung zwischen Sehnervenendigung und Augenmuskelkernen. So weit also aus Thatsachen der physiologischen Anatomie gefolgert werden kann, wäre hier der anatomische Ort des Papillarreflexes zu suchen. Sehr interessant ist in diesem $\mathrm{Zu}$ sammenhang, dass in derselben Sehnervenschicht centrifugale Fasern aus der Sehrinde endigen (Probst, Archiv f. Psychiatrie, Bd. 35). Der optische Reflexapparat ist also in der Lage, von der Sehrinde her dieselbe Beeinflussung zu erfahren wie vom Sehnerven. Diese Beziehung führt zu einem anatomischen Verständniss der corticalen Pupillarreflexe (Piltz) und der Thatsache andererseits, dass bei grosshirnlosen Tauben und bei niedersten Wirhelthieren das Tectum opticum die Function zu übernehmen vermag, welche beim Menschen vom Grosshirn ausgeübt werden. Ganz ähnliche Beziehungen wie zwischen Opticus und Nucleus intratrigeminalis bestehen zwischen Vestibularisendigung und Deiters'schem Kern.

$\mathrm{Zu}$ den Functionen des eben geschilderten optischen Reflexapparates muss es ferner gehören, nach einer peripherischen Netzhautreizung die Stelle des deutlichsten Sehens auf die Ursache dieses Gesichtseindruckes einzustellen. 
1044 30. Wandervers. der Südwestdeutschen Neurologen u. Irrenärzte.

Die Bewegungsempfindungen des Auges werden von dem sensiblen Trigeminuskem der Brücke recipirt and in diesem-nach meiner Ausdrucksweise als "kinästhetische Determinanten aufbewahrt". Auf diesen beruhen die Localzeichen der Netzhaut. Denn die Gegend des sensiblen Trigeminuskernes der Brücke ist mit der Sehnervenendigung im Tectum durch ein ungekreuzt absteigendes "Associationsbündel " verknüpft, nämlich durch in ü n e r's Tractus tecto-pontinus. Es wird nach meiner Auffassung gleichzeitig mit jedem Gesichtseindruck, der durch den äusseren Kniehöcker zur Sehrinde fliesst, via tectaler Selnervenendigung und Tractus tecto-pontinus der sensible Trigeminuskern grereizt und veranlasst, auf dem Wege der medialen Schleife ein Aequiralent des Localzeichens zum Grosshirn zu senden. Aus der Vereinigung dieser und der rein optischen Erregung entsteht das Aequivalent des localisirten Gesichtseindruekes. In ganz analoger Weise habe ich nuch die Localzeichen der Haut verständlich zu machen versucht (Intelligenz und Anpassung, Entwurf zu einer biologischen Darstellung der seelischen Vorgänge, Ostwald's Annalen der Naturphilosophie, Supplementbd. 1903).

Lewandowsky erblickt irrthümlich, wie wir saben, in den grossen Zellen der Sehnervenschicht den Ursprung des prädorsalen Längsbündels. Eher sind sie der Ursprung des Tractus tecto-pontinus und der centralen Haubenbahn. Wo aber entspringt der Tractus Probsti, den Lewandowsky von meinem Nucleus intratrigeminalis ableitete. Ich glaube von den bläschenförmigen Zellen der mesencephalen Trigeminuswurzel selbst, die nach Ramon y Caja I Collateralen an den Kaumuskelkern abgiebt und wahrscheinlich ebenso den Tractus Probsti noch weiter caudalwärts entsendet. Die mesencephale Trigeminuswurzel nimmt ja überhaupt durch die Form ihrer Ursprungszellen und durch diese Collateralen eine ganz einzige und ungeklärte Stellung unter den motorischen Endneuronen ein.

Es ist oben vorausgesetzt worden, dass der in der Eintrittshöhe des Nerven gelegene Trigeminuskern dem Muskelsinn des Trigeminus dient. Dies geht hauptsïchlich daraus hervor, dass aus diesem Kern die Brüclienschleife hervorgeht, die nach Lewandowsky's wichtiger Beobachtung alsbald eine Kreuzung eingeht und sich der medialen Schleife anschliesst. Der sensible Trigeminuskern der Brücke ist. also ein Analogon der Hinterstrangskerne und die in ihn eingehenden Wurzeln entsprechen den Hintersträngen des Rückenmarkes. Die dissociirte Empfindungslähmung bei Lähmung der spinalen Trigeminuswurzel und ihres Kernes beweist, dass diese Gebilde den , cinereopetalen" Reflexcollateralen and dem Grau des'Hinterhornes entsprechen.

Wo aber ist das Analogon der KKl. S. B.? Ich glaube dieses gefunden zu haben. In frontaleren Höhen schliesst sich an den Kern der spinalen V. Wurzel ventral ein Zipfel grauer Substanz an, der mit dem Haupttheil des V. Kernes durch eine schmälere oder breitere Brücke verbunden ist. In diesem Nucleus cerebellaris sieht man aufs deutlichste mediale Fasern des Stricklörpers einstrahlen. Sie durchziehen dabei das Querschnittsfeld der Kl. S. B. Wenn das- 
30. Wandervers. der Südwestdeutschen Neurologen u. Irrenärzte. 1045

selbe in Folge einer Querläsion des Rückenmarkes degenerirt ist ${ }^{\lambda}$ ), so erkennt man dies Verhalten noch deutlicher als am normalen Material.

Dieser Tractus trigemino-cerebellaris degenerirt thatsächlich nich t nach ausgedehnter Zerstörung des Kleinhirns und erscheint, wie ich einer anderen Zwecken dienenden Zeichnung van Gehuchten's ${ }^{2}$ ) entnehme, unter geeigneten Bedingungen nach Verletzung des Strickkörpers in retrograder Degenetion. Er ist also einer von Edinger's Tracti nucleo-cerebellares.

Auch was die Reflexvermictelung anlangt, entspricht der Kern der spinalen Wurzel dem segmentalen Grau des Rückenmarkes. Denn es führen Läsionen des Trigeminuskernes del Oblongata zu Areflexie der Cornea und Conjunctiva. Die zugehörigen Fasern der spinalen Wurzel scheinen besonders leicht lädirbar zu sein, ähnlich wie die Zuleitungsfasern des Patellarreflexes. Daher erkennt 0 p pen heim in der Areflexie der Cornea ein Frübsymptom des Druckes, der auf die Gebilde der Brücke und damit auf die spinalen Wurzeln ausgeübt wird (Neurol. Centralbl. 1905). Die Function der Kl. S. B. anlangend, Jässt sich aus dem klinischen Thatsachenmaterial nur folgern, dass sie für die Leitung des Schmerz-, Temperatur- und kinästhetischen Sinnes entbebrlich ist. Andererseits verdient es Beachtung, dass sie bauptsächlich aus dem Dorsalmark entspringt, welches keinen exogenen Zuwachs zu den Hintersträngen, hingegen die visceralen Schmerzfasern aufnimmt.

Aus anderen Beobachtungen und meinem Falle von acuter Bulbärapoplexie $^{3}$ ) geht hervor, dass das ventrale Drittel des Trigeminuskernes der Oblongata dem Temperatur- und Schmerzsinn eines Ausschnittes aus dem Trigeminusgebiet vorsteht, welcher folgendermaassen begrenzt ist:

Mediale Grenze . . Medianlinie.

Untere $\because \ldots$ Oberlippe.

Laterale $n \quad$. . . Verbindungslinie von äusserem Mund- u. Augenwinkel und deren Fortsetzung nach oben.

Nur dieses selbe centrale Gebiet des Gesicbts wird nach Head von den reflectirten Schmerzen betroffen, welche aus den Organen der Bauch- und Brusthöhle mit Ausschluss des Urogenitalapparates stammen. Auch hierfür giebt, wie ich glaube, die pbysiologische Anatomie der Oblongata eine befriedigende Erklärung:

Es muss trotz des Zweifels hervorragender Neurologen als gesicberte Thatsache gelten, dass der Tractus antero-lateralis ascendens seu Gowersi der Leiter des gekreuzten Temperatur- und Schmerzsinnes ist, wobei die Frage, warum die Hauptmasse seiner Fasern zum Kleinhirn zieht. zunächst allerdings ein ungelöstes Probleu der Kleinbirnphysiologie bleibt. Die Bahn ist im Seitenstrangkern unterbrochen und bat hier Gelegenheit, auf anliegende Gebilde zu irradiiren.

Der zunächst liegende Theil des Trigeminuskernes ist derselbe, der von

1) Auch solches Material verdanke ich der Güte des Herrn Dr. Bickel,

2) Le Névraxe. Bd. IV.

3) Beschrieben von E. Mai, Arch. f. Psych. Bd. 38. 
1046 30. Wandervers. der Südwestdentschen Neurologen u. Irrenärzte.

Herden dieser Gegend mitbetroffen wird, solchen nämlich, die gleichzeitig zu gekreuzter dissociirter Empfindangslähmung von Rumpf und Extremitäten fübren, also in den Tractus Gowersi und den Seitenstrangkern zu localisiren sind. Dibser Thei! des Trigeminuskemes versorgt den oben amschrieberen centralen 'Theil des Gesichts. Erkrankungen der Keimdrüsen irradiiren, Head's zuverlässiger Angabe zufolge, nach der Occipitalzone, die von den obersten Cervicalnerven versorgt wird. Inre Schmerzbahnen dürften also im caudalsten Abschnitt des Seitenstrangkernes eine Unterbrechung erfahren. Auf ähnlichem Wege muss auch mein "Errältungsreflex" zu Stande kommen (Deutsche med. Wochenschr. 1903. 16), nämlich durch Irradiation der Kältebahn im Gowersischen Strang auf den Trigeminus- und Vaguskern.

Herde der genannten Gegend pflegen auch zu Schling- und Stimmlähmung zu führen, woraus alle Autoren geschlossen haben, dass der ventrale Vaguskern mitbetroffen und das Centrum der entsprechenden Vayuswurzeln sei. Dieser Schluss ist hinfällig. Denn, wie an dieser Stelle an Marchi-Präparaten vor zwei Jahren demonstrirt habe, verlassen die Axone des dorsalen Vaguslernes als ventralste Vagusfasern die Oblongata am unteren Pol der spinalen Trigeminuswarzel und durcblieuzen dabei den Ort jener Herde. Die Axone des ventralen Vaguskernes machen die bekannte Schlinge, überkrcuzen am ventralen Rand des dorsalen Graus die Fasern aus dem dorsalen Kern und verlassendie Oblongata als mittlere Vagusfasern, indem sie die spinale V. Wurzel in ihrer ganzen Querschnittsausdehnung durchbrechen. Sie sind mittelst retrograder Degeneration durch v. Gehuchten dargestellt worden ${ }^{1}$ ). Die centripetalen Vagusfasern liegen als echte hintere Wurzeln am woitesten dorsalwärts (Marchi-Degeneration nach Durchschneidung central vom Ganglion jugulare).

Dies sieht man am schörsten etwas caudal rom Eintritt des IX. Nerven, da, wo der sensible Vaguskern seine grösste Ausdehnung erreicht und mit dem Kern der spinalen V. Wurzel verschmilzt. Diese Verschmelanng giebt die Möglichkeit der Irradiation rom sensiblen Vagus auf das Trigeminusgebiet, auf welcher nach meiner Vermuthung die reflectorische Hyperämie der Nasenschleimbäute und der reflectorische Herpes der Hornhaut und der Lippen bei der Menstruation und bei inneren Erkrankungen beruht. Während die Hornhaut im ventralen und im dorsalen Abschnitt des Trigeminusbalbmondes vertreten zu sein scheint, gehört die Mundschleimhaut und der Faupttheil des Hautgebietes Rami III nervi trigemini ausschliesslich den dorsalen zwei Dritteln desselben an und zwar hauptsächlich in frontaleren Höhen. Dies folgt aus dem klinisch-anatomischen Thatbestand bei acnter Bulbärparalyse und seinem Negativ bei Spaltbildungen der Oblongata, die vom dorsalen Grau aus zuweilen ventralwärts vordringen ( L a ehr $\mathbf{r}$, aus Wallenberg's Thierversuchen und schliesslich aus einem hochinteressanten Sectionsfalle von Head und Camp. bell, in welchem eine partielle Erkrankung des Ganglion Gasseri einerseits za Herpeserruption iw Gebiet des dritten Trigeminusastes, andererseits zu

1) Le Nérraxe. Bd. V. 
30. Wandervers. der Südwestdentschen Neurologen u. Irrenärzte. 1047

Marchi-Degeneration der dorsalen zwei Drittel der spinalen V. Wurzel geführt hatte. Aus der Intactheit des sensiblen Kerns der Brücke schliessen die Autoren irrthümlich auf die Zugehörigkeit zum ersten Ast, dessen Projection nach Anderer und unserer Ansicht im ventralsten Theil des Halbmondes liegt. Den sensiblen Kern der Brücke balten wir, wie oben angeführt, für das kinästhetische Centrum des I'rigeminus. Die Controverse über eine etwaige Beimischung von Geschmacksfasern des Trigeminus zum Solitärbündel verliert dadurch an Interesse, dass die grauen Kerne beider sicher mit einander verschmelzen. (Demonstration an Tafeln.)

\section{'Lusammenfassung.}

1. Der Nucleus intratrigeminalis tecti ist der Ursprungskern des prädorsalen Längsbündels.

2. Läsion der spinalen V. Wurzel und ihres Kerns führt zu dissociirter Empfindungslähmung und zu Areflexie der Cornea.

3. Der ventrale Zipfel des Nucleus radio. spin. V, entsendet ein Analogon der KI.-S.-B. kerne.

4. Der sensible V. Kern der Brücke ist das Analogon der Hinterstrangs-

5. Die Kerne des dorsalen X. Kerns treten an ventralen Pol der spinalen V. Wurzel aus und werden durch Läsionen dieser Gegend zerstört.

6. Die in das V. Gebiet reflectirten Visceralschmerzen Head's entstehen durch Irradiation rom Seitenstrangkern auf den Nucleus radicis spinalis V. -

Schluss der Sitzung $5^{1} / 2$ Nachm.

\section{Sitzung: Sonntag, 28. Mai 1905, 9 Uhr Vormittags.}

Vorsitzender: Herr Prof. Grützuer-Tübingen.

Es folgen zunächst geschäftliche Mittheilungen. Als Ort der nächstjährigen Versammlung wird Baden-Baden, zu Geschäftsführern werden die Herren Professoren Krebl (Strassburg) und R. Laquer (Franlifurt) gewählt.

Als Reforatthema wird bestimmt: Dor Stand der Lehre von den vasomotorischen Nerven, und dasselbe Herrn Grützner-Tübingen übertragen.

Herr Edinger theilt mit, es sei der Vorschlag gemacht worden, das Referat drucken zu lassen, vor der Versammlang zu versenden .und in der Sitzung lediglich über dasselbe zu discutiren, um mebr Zeit fürr die Vorträge zli gewinnen.

Herr Fürstner spricht sich gegen diesen Vorschlag aus, da die Wirkungdes gesprochenen und unmittelbar gehörten Wortes doch erbeblich eindrucksvoller und lebendiger sei als der Eindruck, der von der Lectüre zurückbleibt, und weil der wesentiche Werth des Referats gerade darin liege, dass es zum unmittelbaren Meinungsaustausch Gelegenheit gebe.

Herr Erb hält es für besser das Referat zu hören, aber die Leitsätze vorher drucken und zur Vertheilang gelangen zu lassen. 
1048 30. Wandervers. der Südwestdeutschen Neurologen u. Irrenärzte.

Herr Edinger schliesst sich dem Vorredner an, und die Versammlung beschliesst entsprechend diesem Antrag.

Es folgen die Vorträge:

13. Herr Prof. Jelgersma-Leiden: Ueber das Nervensystem der Cetaceen.

14. Herr Privatdocent Dr. Bumke-Freiburg i. B. hat die secundären Degenerationen verfolgt, die nach einer Compression im 5. und 6. Cervicalsegment eingetreten waren. Marchi-Methode, beim Einbetten wurde statt Alkohol Aceton verwandt.

Absteigend waren die Pyramidenbahnen bis ins unterste Sacralmark zu verfolgen, in allen Höhen, besonders deutlich in der Lendenanschwellung waren Fasern zu seben, die durch die vordere Commissur zum gegenüberliegenden Vorderhorn kreuzen. In den Hinterstrüngen war ein schwaler degenerirter Streifen längs des Septums nur durch zwei Segmente nachweisbar, während das Schultze'sche Komma noch nach 10 Segmenten, also in der Höhe der 8. Brustwurzel deutlich erkennbar war. Seine Fasern gingen nicht über in eine dritte Fasergruppe, die dicht unterhalb der Compressionsstelie den dorsolateralen Antheil der Hinterstränge einzahmen. Es ist das jenes Bündel, dessen eigenthümliche Lageänderung zuerst von $\mathrm{Hoche}$ beschrieben ist. In diesem Falle begann die Ueberwanderung dieser Fasern um die hintere Peripherie des Markes in der Höhe der ersten Brustwurzel, sie war vollendet im 4. Lendensegment. Von hier ab rückiten dann die Fasern am Septum entlang nach vorn zur hinteren Commissur. Sie waren noch im Conus nachzuweisen. Eine vierte Gruppe von Fasern endlich lag dicht unterhalb der Compressionsstelle iiber das ganze Hinterstrangsgebiet zerstreat; sie sammelten sich dann im ventralen Hinterstrangsfelde, um schliesslich in der Höhe der 12. Brustwurzel zu verschwinden.

A ufsteigend degrenerirt sind zunächst kurze Bahnen, die sich in den beiden ersten Segmenten oberhalb der Compressionsstelle, also in der Wöhe de: 3. und 4. Halswurzel, über den ganzen Querschnitt vertheilen und die im 2. Cervicalsegment bereits verschwunden sind.

In den Hintersträngen ist bemerkenswerth: das Freibleiben der Bechterew'schen Zwischenzone zwischen Goll'schem und Burdach'schem Strange und eines schmalen Streifens dicht am medianen Septum. Hervorzuheben ist ferner, dass nicht alle Hinterstrangsfasern in den Hinterstrangskernen enden: Einzelne schwarze Ketten ziehen als Fibrae arciformes externae und internae zur gegenüberliegenden und gleichseitigen Kleinhirnseitenstrangbahn, z. Th. die Schleife, z. Th. schon vorher die Pyramide durchsetzend. Das Gowers'sche Bündel giebt Fasern an die graue Substanz des Halsnarks ab; ferner da, wo sich die Kleinhirnseitenstrangbahn vom Gowers'schen Bündel getrennt hat and in den Stricklörper eingetreten ist, Fasern, die in leicht geschwungenem Bogen vom Anterolateraltract zum Corpus restif. ziehen, sodann andere, die in Beziehungen zum Nucleus ambiguus treten, und endlich solche, die, als Fibrae arcuatae ext. um den Strickliörper herum den Deiter'schen Kern erreichen. Schliesslich konnte auch in diesem Falle der Tractus 
30. Wandervers. der Südwestdeutschen Neurologen u. Irrenärzte. 1049

spinothalamicus bis zum centralen Abschnitte des lateralen Sehkügelkernes verfolgt werden.

(Die ausführliche Veröffentlichung wird im Arch. f. Psych. erfolgen). (Autoreferat.)

15. Herr Dr. de Montet spricht über Wanderungen lipoider Substanzen im Centralnerrensystem. Er glauht Myelinstoffe (M. in morphologischem Sinne) als Aufbau-, resp. Reservematerial und als Abbauproduct aus Substanzen mit lipoidem Radical nachgewiesen zu haben.

Myelin findet sich als Aufbaumaterial bei thierischen und menschlichen Embryonen und Foeten. in Form von feinen, mit „Neutralroth primär nicht färbbaren Kömehen, die mehr, diffus in den Gefässwänden und Bindegewebszellen der Pia vertheilt sind; später in den Zellen angebäuft auch zwischen den Nervenzellen. (Markscheidenbildung?) Bei Neonatis ganz älnlich. - Auch bei Kindern sind diese Gebilde in den ersten Lebensjahren vorwiegend ungerärbt; es zeigt sich eine deutlichere Gruppirnng in Adventitialund Bindegewebszellen.

Es werden dann die Gründe für die Deutung als Aufbaumaterial angefürt, zum Theil entgegen den Zappert'schen Anschauungen, aber in Uebereinstimmung mit Wlassak.

Als schon physiologisch bei Mensch und Thier vorkommende Abbauproducte treten diese Myelinstoffe in Form meist primär mit Neutralroth färbbarer Körner und Schollen auf. (Ursache dafür: Färbbarkeit des Myelins bei Kernuntergang. Farbstoffspeichernde Eigenschaften der künstlichen Markscheidenderiate, event. noch andere Factoren).

Belege für diese Ansicht sind: die hochgradige Vermehrung im Senium, bei chronischen Erkrankungen (Phthise, Diabetes-Parallelismus nuit dem Ernährongszustand); bei hochgradigen Infectionskranliheiten (Typhus), bei eigentlichen Gehirnprocessen (Meningitis) mit auffallend grossen Mengen; für Urämie und Strychninvergiftıng auch experimentell am Thier nachgewiesen; ebenfalls sehr starke Vermehrung bei Atrophie, Hydrocephalus, Epilepsie, progressiver Paralyse).

Im gleichen Sinne spricht die experimentelle Erzeugung am Thier, durch allgemeine Schädigung des Gehirns, ebenso die Befunde am peripheren Nerven, im Extrem bei Durchschneidung. (Roth färbbare Schollen im Verlauf der Markscheiden; Körnchenzellen mit Neutralroth intensiv färbbaren Granulis.)

Der Transport dieser Stoffe, die nicht autochthon in der Pia entstehen, geschieht vornehmlich auf dem Lymphweg, in fester und wahrscheinlich auch in flüssiger Form. Die Aufnahmefähigkeit der Adventitialzellen, Endothelien und Ependymzellen auch für feste Körper wurde experimentell nachgewiesen. (Intracerebrale Injectionen.)

Endlich wird die Vermuthung einer gemeinsamen Rolle beim Fettabbau zwischen diesen grobgranulirten Adventitialzellen, eigentlichen Mastzellen und nicht metachromatischen, klasmatocytenartigen, feingranulirten Zellen ausgesprochen.

16. Herr Dr. A. Homburger-Frankfurt a. M. demonstrirt eine Anzahl 
1050 30. Wandervers. der Südwestdeutschen Neurologen u. Irrenärzte.

von Original-Neurogliapräparaten Weigert's, die sich vorzugsweise auf dessen letzte Mittheilungen über Kleinhirnveränderungen bei Tabes, progressiver Paralyse und Syphilis beziehen. Die Objecte zeigen deutlich die herdförmigen Sklerosen der Rinde, welche hauptsächlich aus massenhaft neugebildeten Bergmann'schen Radiärfasern bestehen; daneben sind Ansätze zur Production einer gliösen Randschicht tangentialer Richtung bemerlibar; sehr ausgesprochen ist die Sklerose der Marksubstanz häufig bei Paralyse und Lues. Diese Veränderungen treten bei allen drei Krankheiten in wechselnder Intensität, aber mit grosser Häufigkeit auf und zeigen überall den gleichen Charakter. Schnitte vom tabischen Rückenmark, sowie normale Präparate von Pons, Oblongata und Rückenmark, sowie von Sklerosen der Marksubstanz bei progressiver $\mathrm{Pa}$ ralyse lassen die Fortschritte erkennen, die Weigert besonders in der Darstellung der feinen Fasern gemacht hat; über die von jhm vorgenommenen Aenderungen der Methode hat er keine Mittheilungen hinterlassen.

Homburger bespricht sodann die Gründe der mangelhaften Haltbarkeit der nach Weigert's Angaben hergestellten Präparate und weist besonders auf die bleichende Einwirkung reducirender Gase, Formaldehyd, $\mathrm{SO}_{2}, \mathrm{H}_{2} \mathrm{~S}$, besonders aber des Leuchtgases hin, und warnt deshalb vor der Aufbewahrung yon Neuroglia- und sonstigen mit Metbylviolett gefärbten Präparaten (Fibrin. Bakterion) im Laboratoriumsraum. Vortragender, dem die Bearbeitung der Weigert'schen Sammlung übertragen wurde, stellt spätere ausführliche Mittheilung der besonderen Ergebnisse in Aussicht.

17. Herr Dr. F. Blum: Ueber die specifische Behandlung des Morbus Basedowii.

18. Herr Dr. van Oordt-St. Blasien i. Schw.: Therapeutische Erfahrungen bei der Basedow'schen Krankheit.

Der Anschauung von der thyreogenen Aetiologie der Basedow-Krankheit verdanken wir eine Anzahl als specifisch geltender Behandlungsmethoden. Es sind dies die Strumectomie, die Behandlung mit Thyreoidin und Jodothyrin, mit Thyreoidserum Möbius-Merck, mit Rodagen, mit dem Serum thyreoidisirter Kaninchen, Hunde und Ziegen, mit der Milch thyreoidectomirter Thiere nach Lanz and Göbel, und den Tabletten aus dem Blnte thyreoidectomirter Ziegen nach Madsen. Verfasser hat 18 Fälle beobachtet, die nach einer oder mehreren der vorgenannten Methoden behandelt sind. Die Strumectomie setzte in 4 Fällen die Pulsfrequen h herab, und liess in 2 derselben die orweiterten Herdgrenzen zurückgehen unter Hebung des Körpergewichtes und Besserung des psychischen Verhaltens. Vom Thyreoidin sah er keinen Erfolg; bei 5 mit Thyreoidserum Behandelten ist subjective Besserung in 2 Fällen erzielt worden und die Pulsfrequenz herabgesetzt, die Struma nur in einem Falle etwas beeinflusst worden. Etwas besser sind die Erfolge mit Rodagen. Die Madsenschen Tabletten wurden erfolglos genommen. Die Lanz'sche Milch schaffte vorübergehende Besserung des Allgemeinbefindens während des Gebrauches. Alle 18 Kranke sind auch heute noch als Basedow-Kranke anzusehen. Vortragender stellt in einer Statistil seiner während der letzten 10 Jahre behandelten 66 Basedow-Fälle fest, dass die Behandlungsresultate unter der elektro- 
30. Wandervers. der Südwestdeutschen Neurologen u. Irrenärzte. 1051

therapeutischen, klimatischen, physikalisch-diätetischen und sedativen Behandlung mindestens gleich günstig waren, und empfiehlt auch fernerhin diese Therapie in den Vordergrund zu stellen bei gleichzeitigen Behandlungsversuchen mit einem der oben genannten, sogenannten Specifica, besonders mit dem Rodagen. Gute Erfolge sah er auch von periodischer Application eines Eisbeutels auf die Struma. Die Strumectomie ist nur in rasch progressiven und solchen Fällen zu versuchen, die sich erfolglos der neurologischen Behandlung anvertraut haben oder aus socialen Gründen nicht unterziehen können.

19. Herr Dr. R. L. Schütz (Wiesbaden): Ueber eine schwere Form von chronischem Colonspasmus.

Schütz hat in den lotzten zwei Jahren bei zwei Mädohen und einer Frau im Alter von 19-22 Jahren eine schwere Form von chronischem Colonspasmus beobachtet, wie sie bisher nicht beschrieben worden ist.

Der Colonkrampf dauerte zur Zeit der Beobachtung 5-7 Wochen und war mit völliger Verstopfung und den heftigsten Schmerzen verbunden. Die Kranken boten ein Bild schwersten Krankseins. Der Spasmus ist bei zwei Patientinnen, die Schütz weiter verfolgen konnte, seither noch häufig aufgetreten und hat bei der einen zur Entfernung des Processus vermiformis Anlass gegeben, ein Eingriff, der ohne jeden Erfolg blieb.

Das eigenartige Krankheitsbild - ein Zustand maximaler Contraction des Colon, die ohne Unterbrechung wochenlang besteht - wird verständlich im Hinblick anf die Bleikolik, nur dass es sich nicht um die Wirkung eines von aussen eingeführten Giftes, sondern um eine abnorme Erregbarkeit des Darmnervensystems handelt. Vortragender bespricht kurz Pathologie, Diagnose, Prognose und Therapie.

Der Vortrag erscheint demnächst im Archiv für Verdauungskrankheiten. Idiotie.

20. Herr Prof. Dr. Weygandt (Würzburg): Dementia praecox und

Bei einer Reihe von Fällen der Dementia praecox zeigt die Vorgeschichte imbecille Veranlagung von Jugend auf; es handelt sich nicht nur um eine sogenannte Pfropfhebephrenie, sondern auch eine typische Katatonie und paranoische Demenz kann sich auf imbeciller Basis entwickeln.

Daneben wurden' seit Langem bei tiefstehenden Idioten bizarre Körperhaltungen, Grimassiren, rhythmische Bewegungen, sinnlose rhythische Sprachäusserangen und ähnliche segenannte Tics beobachtet, auch ein ablehndes oder negativistisches Verhalten, sowie Andeutungen von Befehls-Automatie; bekannt ist weiterhin vielfach die auffallend schwache Entwicklung der affectiven Sphäre, die ja mit den Anlass zur Aufstellung des Begriffes der Moral insanity gegeben hatte. Allerdings decken sich die letzteren Fälle nicht mit den durch jene Tics charakterisirten.

Kraepelin suchte neuerdings die Idioten mit unzugänglichem störrischem Wesen, Haltungsstereotypien, Manieren, Schrullen, rhythmischen Bewegungen u. s. w. als Frühform der Dementia praecox aufzufassen. Indess lässt sich bei eingehender Analyse zahlreicher Fälle von Ldioten mit solchen Tics gewöhnlich feststellen, dass ihrem Defect eine bestimmte cerebrale Ursache 
1052 30. Wandervers. der Südwestdeutschen Neurologen u. Irrenärzte.

zu Grunde liegt, sowohl Entwicklungshemmung wie auch früh einsetzende Hirnentzündungen, Porencephalie, Hirnatrophie, chronische Meningitis, Hydrocephalie, auch Meningismus. Es sind in solchen Fällen die Tics viel unge$z$ wungener ebenso wie der idiotische Blödsinn durch eben diese verschiedenen, die Hirnentwicklung störenden Ursachen zu erklären.

Gerade beilmbecillen, aus denen sich die sogenannten Pfropfhebephrenien rekrutiren, finden sich Tics verbältnissmässig selten.

Es ist weiterhin zu betonen, dass solche Tics keineswegs pathognomonisch für Dementia praecox sind, sondern auch bei andern Psychosen wie Paralyse, Epilepsie u. s. w. vorkommen können.

Ferner wurden auch Cebergänge von Imbecillität zum manisch-depressiven Irresein, die Vereinigung von Imbecillität mit Hysterie sowie das Auftreten von Paralyse auf imbeciller Basis beobachtet.

Schliesslich ist zu betonen, dass Andeutungen jener ticartigen Symptome auch auf früher Stufe der normalen Kindesentwicklung zu beobachten sind, negativistische and Echo-Symptome, Grimassiren, gezwungene Haltungen, rhythmische Bewegungen und selbst sprachliche Aeusserungen, die sich von denen der Dementia praecox kaum unterscheiden.

Es handelt sich also bei jenen Tics der Idioten keineswegs um Symptome einer früh einsetzenden Dementia praecox, wohl aber um Zeichen, die die Pathogenese der Dementia praecox selbst beleuchten können, insofern sie sich darstellen als ein Rückfall auf eine Frühstufe der Kindesentwicklung, in der als erste Vorstufe, der Zweckhandlung Iediglich eine Auslösung psychomotorischer Antriebe in der stimmlich-sprachlichen, wie in der sonstigen muslsulären Sphäre stattfindet.

21. Herr Dr. L. Roemheld (Schloss Hornegga.N.): Ueber den Korsakow'schen Symptomenkomplex bei Hirnlues.

Das Korsakow'sche Syndrom, das im Wesentlichen aus hochgradiger Störung der Merkfähigkeit bei relativ gutem Erinnerungsvernögen für die Vergangenbeit, aus zeitlicher und örtlicher Desorientirtheit und Neigung zu Fabulationen und Pseudoreminiscenzen besteht, ist bis jetzt bei Hirnlues noch nicht beobachtet worden.

R. berichtet über einen Fall von Hirnlues bei einer 46jährigen Dame. Somatisch fand sich: Druckempfindlichkeit der linken Schädelhälfte, fast ganz erloschene Pupillenreaction, Schlaffheit der rechten Gesichtshälfte, Abweichen der Zunge nach rechts, klonische Zuckungen der rechten Gesichtshälfte und bisweilen auch der rechtsseitigen Extremitäten, Staungspapilie links mehr als rechts, $\mathrm{S} \mathrm{I}=4 / 8$, r. $4 / 4$, Aufgehobensein des rechten Patellarreflexes während er links schwach war. Dazu kamen vorübergehend Blasen- und MastdarmIncontinenz. Das psychische Verhalten zeigte einen Stupor wie man ihn bei Hirntumoren bisweilen findet, ferner hochgradige Störung der Merkfähigkeit für optische und akustische Reize bei ziemlich gutem Erinnerungsvermögen für die Vergangenheit, vollständige örtliche und anfangs auch zeitliche Desorientirtheit. Die Kranke fabulirte viel, theilweise in phantastiseher Weise, theils in Form von sog. Verlegenheitsfabulationen. Das intellectuelle Ver- 
30. Wandervers. der Südwestdeutschen Neurologen u. Irrenärzte. 1053

halten der Patientin zeigte keinen gröberen Defect. Auf Schmiercur keine Besserung, wohl aber auf intensive subcutane Jodipinanwendung. Völliges Schwinden der psychischen Störung. Zurüok blieb ein Erinnerungsdefect, der sich auf $3 / 4$ Jahre erstreckte. Somatisch blieb eine gewisse Neigung zu leichten Schwindelanfällen und Abblassung der Papillen zurück.

Da Alkoholismus bei der Patientin ausgeschlossen ist, dürfte die Hirnlues, die sich pathologisch-anatomisch wohl theils als diffuse arteritische Erkrankung, theils als ein in der linken Hemisphäre cortical bez. subcortical sitzendes Gumma manifestirt hat, Ursache des Korsakow'schen Syndroms gewesen sein. Ob die intracranielle Drucksteigerung oder die syphilitische Intoxication speciell die psychisohe Störung bedingt haben, lässt Verfasser unentschieden, neigt sich aber eher der letzteren Auffassung zu.

22. Herr Dr. L. Link, Privatdocent (Freiburg i./B.): Ueber den Babinski'schen Reflex.

Vortr. theilt Untersuchungen mit über das Auftreten dieses Reflexes bei Injectionen von Scopolaminum hydrobromicum, die zu Zwecken der allgemeinen Narkose oder als Sedativum für Geisteskranke angewendet wurden. Der Babinli'sche Reflex fand sich bei 31 von 36 Fällen; bei den fünf negativen waren 2 Patienten, die auch sonst ganz oder theilweise sich refractär erwiesen, 3 hatten nur kleine Dosen, 0,00045 und dann in Abständen von einer oder mebreren Stunden 0,00015 erhalten und warden erst 21/2, 4 und 15 Stunden nach der ersten Injection untersucht. Bei allen ausser bei sechs Patienten, die aber auch keinerlei Zeichen einer nervösen Erkrankung, welche Babinsk'sches Phänomen bedingt, darboten, wurde vor oder nach der Narkose festgestellt, dass sie normalen Beugetypus der Zehen hatten. Die kürzeste Zeit für das Auftreten dieses somit bei Scopolamin-Injectionen fast constanten Reflexes - in der Literatur wird sein Auftreten nur von Kutner und Volkmann nebenbei erwähnt - war einige Minuten nach Injection von 0,0012, die kleinste Dosis, nach der er beobachtet wurde, 0,0004. Das meist gleichzeitig injicirte Morphium veranlasste den Reflex sicher nicht, wie zweifelloses Auftreten desselben nach Injection von Scopolamin allein und das Vorhandensein eines lebbatten normalen Plantarreflexes in einem Fall schwerer Morphiumvergiftung bewiesen. Die zur Anwendung kommenden Dosen waren 0,0012 bis 0,002 als Einzeldosis, 0,0012 mit zweimaliger Wiederholung und 0,00045 und dann mehrfach 0,00015 .

Vortr. weist auf das auffallende Missverhältniss hin, das bestand in der meist vorhandenen Steigerung der tiefen Reflexe - öfters Fussclonus - und der von ihm und anderen Autoren beobachteten Herabsetzung des Muskeltonus; lediglich als Theilerscheinung einer allgemeinen Reflexsteigerung kann somit der Babinski'sche Reflex hier nicht aufgefasst werden. - Bei 6 Fällen konnte constant das Verschwinden des durch Scopolamin hervorgerufenen Babinskischen Reflexes unter dem Einfluss von wenigen Cabikcentimetern Chloroform nachgewiesen werden. $14 \mathrm{mal}$ fand sich nur beim Streichen der Fusssoblen Dorsalfexion der grossen Zehe, beim Stechen dagegen normale Plantarflexion; das umgekehrte Verhalten wurde nie beobachtet. 
1054 30. Wandervers. der Südwestdeutschen Neurologen u. Irrenärzte.

Vortr. hält das Phänomen für bedingt durch die functionelle Ausschaltung der Grosshirnrinde durch das Scopolamin, entsprechend dem Auftreten des Babinski'schen Reflexes im tiefen physiologischen Schlaf and im epilepti-' schen Coma.

Der Vortrag erscheint in extenso im Neurologischen Centralblatt.

(Autoreferat).

23. Herr Dr. Bayerthal (Worms): Ueber Spätmeningitis nach Schädelverletzungen.

Die Fälle, in denen der ärztliche Sachverständige sein Gutachten darüber abzugeben hat, ob eine Hirnhantentzündung als Folge eines Schädelbruches aufzufassen ist, sind verhältnissmässig hänfig und bereiten der Beurtheilung meist keine Schwierigkeit.

Weniger gilt dies für die auffallenden und ausserordentlich seltenen Fälle, in denen die Kranken längere Zeit nach scheinbar vollständiger Wiederherstellung an einer Meningitis zu Grunde gehen. Freilich wird auch hier, sobald sich bei der Section Hirnabscesse traumatischer Herkunft finden, die Deutung des ätiologischen Zusammenhanges keinen Schwierigkeiten begegnen.

Wie verhält es sich aber in Fällen, wo diese Infectionsquelle nicht nachweisbar ist und zwischen Schädelfractur und Meningitis bereits Monate oder Jahre verflossen sind? Zur Beschäftigung mit dieser Frage gab Vortragendem der folgende Fall Veranlassung:

26jähriger Tünchergeselle, bis zu einer am 27. April 1901 erlittenen Basisfractur stets gesund. Auf Grund von Schwindelanfällen, die auf eine Labyrinthläsion zurückgeführt wurden, war eine Rente von $50 \mathrm{pCt}$. bewilligt worden. Bei der Nachuntersuchung am 8. August 1902 hlagte der Verletzte über häufig auftretende Kopfschmerzen ohne bestimmite Localisation und über eine a uffallende Abnahme seines Gedächtnisses. Am 21. September 1903 acut einsetzende. Meningitis, der Patient nach 4 Tagen erlag. Die Section und mikroskopische Untersuchung (Nissl) ergaben das typische Bild der eitrigen Hirnhautentzündung. Benachbarte Eiterherde oder entfernte Infectionsherde, von denen bacterielle Krankheitserreger in das Gehirn hätten gelangen können, konnten nicht nachgewiesen werden - das Interesso des Falles beruht auf dem ursächlichen Zusammenhang der Meningitis mit der beinahe $21 / 2$ Jahre zurückliegenden Schädelfractur. Vortragender nimmt an, dass bereits vor dem Einsetzen der Meningitis histologische Schädigungen im Gehirn bestanden haben, welche mit dem erlittenen Unfall in Zusammenhang zu bringen und als Locus minoris resistentiae gegenüber den auf dem Wege der Blut- und Saftbahnen eingedrungenen Infectionserregern aufzufassen sind. Diese Annahme sei berechtigt, weil Gedächtnissdefecte, wie der in obigem Falle vorhandene, sich nicht selten nach Basisfracturen entwickeln (Graf, König) und auf eine Erkrankung des Gehirngefässapparates, möglicherweise auch der Ganglienzellen zurückgeführt werden müssen. Die Frage, welche mikroskopischen Veränderungen des Gehirns das letztere zu einem Locus minoris resistentiae gegenüber Mikroorganismen und ihrer Toxine machen, wird im Sinne Schultze's und Erb's (Curschmann's) beantwortet. Dem Gutachten des 
30. Wandervers. der Südwestdeutschen Neurologen u. Irrenärzte. 1055

Vortragenden entsprechend, hat die Berufsgenossenschaft den Hinterbliebenen des Verletzten die gesetzliche Rente bewilligt.

24. Herr Dr. L. Merzbacher (Heidelberg): Das Auftreten von Lymphocyten in der Spinalflüssigkeit nach syphilitischer Infection.

Es wurden 26 Krunke (zumeist aus der Heidelberger Irrenklinik) punetirt, die mit grosser Wahrscheinlichkeit sich syphilitisch inficirt hatten und die zur Zeit der Untersuchung frei geblieben waren von allen Zeichen, die auf eine sogenannte organische Erkrankung des Nervensystems zurückgeführt werden können. Vier Kranke - sie werden einer besonderen Gruppe zugerechnet - allerdings zeigten gewisse Läsionen des Centralnervensystems, doch diese Affectionen machen an und für sich erfahrungsgemäss keine Lymphocytose. - Von den 26 Kranken, bei denen allen mit Sicherheit der Verdacht einer beginnenden Paralyse oder Tabes aus dem klinischen Verlauf der Erkrankung ausgeschlossen werden konnte, boten 23 einen positiven Befund, d. h. durch die Lumbalpunction konnte eine in vielen Fällen recht beträchtliche Vermehrung der zelligen Elemente in der Cerebrospinalflüssigkeit aufgedeckt werden. 2 Kranke hatten einen zweifelhaften Befund, eine Kranke einen negativen; mit Abzug dieser 3 Fälle war also der Befund in 89,7 pCt. der Fälle positiv. Die Kranken litten an den verschiedensten Geisteskrankheiten (circuläres Irresein, Dementia praecox, Arteriosklerose etc., 2 waren Jandstreicher, 5 Puellae).

Verfasser vergleicht seine Befunde mit denen anderer Untersucher; aus der kritischen Verwerthung der Gesammtheit der Erfahrungen ergeben sich Deductionen, die geeignet erscheinen, die Haltlosigkeit der Lehre von der $\mathrm{Me}$ ningitis oder der meningitischen Reizung als Ursache der Lymphocytose zu demonstriren. Bei dem heutigen Stande unserer Kentniss kann man nur soviel aussagen, dass die syphilitische Infection als ein Noxe zu betrachten ist, die unabhängig von den klinischen Symptomen der Syphilis eine Hyperlymphocytose erzeugt durch uns noch unbekannte Alterationen des Mechanismus, durch dessen Thätigkeit die Lymphocyten in die Cerebrospinalflüssigkeit gelangen.

Eine ausführliche Mittheilung erscheint demnächst im Centralblatt für Nervenheilkunde und Psychiatrie (Gaupp). (Autoreferat.)

45. Herr Privatdocent Dr. F. Jamin (Erlangen): Ueber Hautreflexe an den Armen.

Die oberen Extremitäten sind im Vergleich zu den unteren auffallend unempfindlich in Bezug auf Hautreflexe. Bei erhaltener willkürlicher Beweglichkeit der Arme treten auf schmerzhafte Reize an der Hand Abwehrbewegangen auf, bei Lähmungen, insbesondere bei der Hemiplegie, sind in der Regel keine dentlichen Hautreflexe an den Armen zu erzielen. Neuerdings hat Oppenheim einen Pronationsreflex bei der Diplegia spastica der Kinder. beschrieben, C. Reuter eine ieflectorische Streckbewegung bei einem Paralytiker.

Vortragender hat einen pathologischen Hautreflex am Arm Jabre lang, 
1056 30. Wandervers. der Südwestdeutschen Neurologen u. Irrenärzte.

zuerst 1902, bei einer vollkommen paraplegischen Kranken beobachtet, bei der anfänglich nur der rechte Arm eine hochgradige Parese vorwiegend der Strecker mit Beugecontractur im Ellenbogengelenk und in den Fingergelenken zeigte. Anf tiefen Stich in die Hoblhand oder, in die Fingerspitzen oder auf energischen Druck an einer Fingerluppe erfolgte mit der schmerzhaften Empfindung zunächst eine kurze Abwehrbewegung im Sinne der fast ausschliesslich möglichen Beugung des Arms uud der Finger. Dann aber trat, etwas verspätet, eine langsame nicht willkürlich zu unterdrückende Streckbewegung ein: Abduction im Schultergelenk und Einwärtsrollung des Armes, Streckung im Ellenbogengelenk, Pronation und Volarflexion der Hand, Streckung und Spreizung sämmtlicher Finger mit Einschluss des Daumens. Nach einigen Secunden kehrte der Arm in die Beugecontracturstellung wieder zurück.

2 Jahre später war der rechte Arm vollkommen gelähmt, die Sensibilität blieb zienlich gut erhalten, die Sehnenreflexe fehlten an Armen und Beinen ganz. Der Streckreflex auf starken Stich in die Hohlhand war gleichwohl immer noch in gleicher Ausdehnung regelmässig auszulösen und zeigte sich jetat auch am linken Arm, der nunmehr, wie früber der rechte, ebenfalls Schwäche der Strccker und Contractur der Beuger aufwies. Trotz des Fehlens der Sehnenreflexe blieb bis zum Tode ausgesprochene Hypertonie in beiden Armen bestehen, ebenso in den Beinen, die gleichfalls lebhafte Hautreflexe mit Babinki'schem Zehenphänomen und Oppenheims Unterschenkelreflex zeigten.

Bei der Anfangs dieses Jahres vorgenommenen Autopsie wurde eine ausgedehnte multiple Sklerose mit völliger Entmarkung des Brustmarks und des oberen Halsmarks gefunden. Nur im unteren Halsmark und in einem Theil des Lendenmarks sind noch markhaltige Reste des Vorderseiten- und des Hinterstrangs erhalten. Die bulbäre Kerngegend ist nahezu frei ron Herden, ausgedehntere Sklerosen bestehen in der linken Hemisphäre in der Gegend des Linsenkerns und der, inneren Kapsol. $D_{a}$ aber in den marklosen Partien fast durchweg noch wohlerhaltene Axencylinder nachweisbar sind, lässt sich die eigenartige, pathologische Reflexbewegung aus dem anatomischen Befund nicht mit Sicherheit deuten. Aehnliche Streckreflexe an den Armen auf sehr schmerzhafte und anbaltende Reize - nicht auf Kälte - und nur selten auf Strichreize - waren nur noch in zwei weiteren Fällen mit dem klinischen Bilde einer fortgeschrittenen multiplen Slilerose zu finden. Auch waren sie nachweisbar bei einem im Coma eingelieferten Pneumoniekranken neben schwachen Sehnenreflexen, leichter Hypertonie der Glieder, Babinski'schem und Oppenheim'schem Zeichen und dem ron Oppenheim angegebenen Fressreflex. In diesem wenige Stunden nach der Untersuchung letal verlaufenen Falle fand sich autoptisch nur ziemlich hochgradiges Oedem des Gehirns.

(Eigenbericht).

26. Herr Privatdocent Dr. M. Rosenfeld (Strassburg): Ueber Partial defecte bei Katatonie.

R. berichtet über Endzustände von Katatonie, in denen festgestellt werden konnte, dass die Kranken die Fähigkeit verloren hatten, durch Betasten Gegen- 
30. Wandervers. der Südwestdeutschen Neurologen u. Irrenärzte. 1057

stände zu erkennen. Also eine Störung, welche man bei organischen Erkrankungen der Rinde und bei Rindenverletzungen als Tastlähmung zu bezeichnen pflegt. Diese Störung im Reiche des Tastsinnes wurde nur dann als sicher erwiesen betrachtet, wenn die Kranken die Prüfung auf die einfachen Tastempfindungen obne jede Störung an sich vornehmen liessen, correcte, positive Antworten gaben und die sogenannten einfachen Empfindungsqualitäten, also Berührungsempfindung, Temperatursinn, Lageempfindung, Ortsinn und Drucksinn sich intakt erwiesen.

Man wird aus den an anderer Stelle mitzutheilenden Krankengeschichten entnehmen können, dass die Patienten zur Zeit der Prüfung genügend aufmerkten und dass vor allem nicht Negativismus und Vorbeiantworten die Antworten der Patienten beeinflusste. Die Kranken befolgten alle während der Untersuchung an sie gerichteten Aufforderungen, gaben positive Antworten, bezeichneten die von einem Reiz getroffenen Hautstellen durch Hindeuten mit der anderen Hand oder durch Bewegung der betreffenden Finger. Sie benannten die Gegenstände, falls sie den einen oder den anderen richtig erkannten, mit dem richtigen Namen. Mit dem Gesichtssinne wurden alle Objecte sofort richtig erkannt und benannt. Es liegt also zunächst kein, Grund vor, Schwankungen der Aufmerksamkeit, Negativismus und Vorbeireaction für das Zustandekommen jenes Ausfalls verantwortlich zu machen. Die Thatsache, dass die Störung im Wiedererkennen von Gegenständen in einem Falle nur halbseitig bestand, wird auch für die Richtigkeit dieser Annahme sprechen. Das Wiedererkennen von Gegenständen durch Betasten verlangt zum Theil wenigstens complicirte associative Verknüpfungen einfacher Tastempfindungen, unter welchen das Lagegefühl und Muskelbewegungsvorstellung vielleicht die Hauptrolle spielen.

Lissauer hat seiner Zeit schon die Behauptung ausgesprochen, dass die Fähigkeit, Gegenstände durch Betasten zu erkennen, mebr Intelligenz, d. h. complicirtere associative Leistungen erfordern, als das Wiedererkennen durch andere Sinne.

Daun wäre also jener Ausfall auf dem Gebiete des Tastsinnes ein Symptom von Demenz, ein Partialdefect, von dem es nur noch fraglich erscheint, ob er für bestimmte Formen der katatonischen Demenz charakteristisch ist. Ich habe noch eine ganze Reihe von dementen Kranken auf diese Störung im Bereiche des Tastsinnes untersucht und fand dieselbe nur noch in zwei Fällen von Paralyse mit leichten Anfällen. Sonst nicht. Selbst in einem Fall von hochgradiger seniler Demenz mit vollständiger Aufhebung des Gedächtnisses und der Merkfähigkeit fehlte die Störang.

Es ist nun sehr auffällig, dass in den vier Fällen von Katatonie, in welchen sich die Störung im Bereiche des Tastsinnes fand, primäre Störungen der psychomotorischen Innervationsverhältnisse lange Zeiten hindurch bestand und das Krankheitsbild beherrschte. Darnach könnte es scheinen, als wenn die beschriebenen Partialdefecte im Gebiete des Tastsinns $\mathrm{za}$ den motorischen Störungen der Katatonie in Beziehung stehen und nur mit den letzteren zusammen auftreten. 
1058 30. Wandervers. der Südwestdeutschen Neurologen u. Irrenärzte.

27. Herr Dr. Pfersdorff (Strassburg i. E.): Die Prognose der De mentia praecox.

Auf Grund der Katamnese von 150 Fällen, welche von 1890-1900 zum ersten Male in der Strassburger Klinil beobachtet wurden, liessen sich folgende Verlaufsarten der Dementia praecox feststellen:

1. 110 Fälle, die im acuten Stadium und in der Remission katatonische motorische Reizerscheinungen boten.

In 75 Fällen trat keine Remission auf. Diese Kranken blieben dauernd internirt. In 23 Fällen schob sich nach dem ersten Anfall eine mehrjährige (von 2 bis 10 Jahien) Remission ein. Während derselben finden sich die motorischen Reizerscheinungen des acuten Stadiums, sie sind jedoch weniger intensiv und treten phasenweise auf. Eine weitere Gruppe zeichnet sich dadurch aus, dass die Kranken oft Beschäftigung und Aufenthaltsort wechseln. Eine Anzahl Kranker konnte so für kürzere Zeitabschnitte Berufsarbeit leisten, je nach dem Stande der Intelligenz; das Sinken derselben tritt in dieser Gruppe nicht in den Vordergrund. Die Intelligenz war intact in sechs Fällen; im Verlaufe dieser traten nach dem ersten Anfall periodisch Phasen von mehrtägiger Dauer auf, in denen die Kranken reizbar und negativistisch sind; sie leisten volle Arbeit. Kein Recidiv bei 4-12jähriger Beobachtung.

$\mathrm{Zu}$ dieser ersten katatonischen Hauptgruppo sind noch Fälle zu rechnen, in denen sich im Verlanfe mehrerer Jahre ohne acute Reizerscheinungen, langsam eine Charakterveränderung entwickelt. Die Demenz giebt sich vorwiegend in der Abnahme der Intelligenz kund. Die Interesselosigkeit beruht hier auf einer Einbusse an Vorstellungen. Auch hier sind periodische Erscheinungen vorhanden.

Die 2. Hauptgruppe (40 Fälle) ist dadurch ausgezeichnet, dass die motorischen Reizerscheinungen weniger ausgeprägt sind, dass die Schwankungen des Affectes das llinische Bild beherrschen. Die Schwäche des Affectes ist für diese Zustände charakteristisch.

In 12 Fällen entwickelte sich die Demenz nach einer grösseren Anzahl meist nicht lang'e dauernder Depressionen. Die Demenz giebt sich kund in vollständiger Gleichgültigkeit bei erhaltenem geistigen Besitzstand. Trotz erhaltener Vorstellungen fehlt das Interesse. Diese Schwäche des Affectes zeigt sich auch in der Labilität der Stimmungslage. Bei diesen Fällen findet sich auch noch gezierte Sprechweise, Vorliebe für Sprüche und für Redensarten.

In 12 Fällen traten ebenfalls zahlreiche Depressionszustände auf. Dieselben sind dadurch bemerkenswerth, dass auf den geringsten Reiz eine starke Affectreaction erfolgt. Die begleitenden excessiven mimischen Bewegungen drücken der $\Delta$ ffectäusserung den Stempel des Läppischen auf. Die Stimmung ist meist leicht depressiv, jedoch stets äusserst labil. Die Kranken produciren zahlreiche Wahnideen und Sinnestäuschungen. Der terminale Vorstellungsverlust ist nicht bedeutend, die Kranken werden jedoch durch die continuirlichen Affectschwankungen an einer geregelten Beschäftigung verhindert.

In $8 \mathrm{Fällen}$ bestand dauernd motorische Erregung, mit begleitender Euphorie. Stimmungsw echsel und Beeinflussbarkeit fehlt. Im Laufe der Jahre 
30. Wandervers. der Südwestdeutschen Neurologen u. Irrenärzte. 1059

lassen diese Reizsymptome nach; die Erregung änssert sich dann in bastigem Sprechen und Reizbarkeit. Die Kranken sind dauernd arbeitsfäbig. In den Verlauf schieben sich Depressionen, Zustände motorischer Gebundenheit ein.

Kein Intelligenzdefect. Die letzte Gruppe, 8 Fälle, ist dadurch ausgezeichnet, dass dauernd eine leichte Euphorie ohne sonstige Reizerscheinungen besteht. Stärkere Affectreactionen können nicht mehr stattfinden. Die Kranken schildern sich selbst als "kalt".

Sie leisten ihre regelmässige Arbeit und baben Interesse für diese. Periodische Symptome fehlen vollkommen. Recidive wurden nicht beobachtet, völlige Heilung wurde nicht vorgefunden. $16 \mathrm{pCt}$. der Kranken waren dauernd arbeitsfähig.

Schluss der Sitzung $121 / 4$ Uhr.

Freibarg und Frankfurt, im Juni 1905.

Bumke. Homburger. 